\title{
Automorphisms and Quasi-Free States of the CAR Algebra
}

\author{
N. M. Hugenholtz \\ Instituut voor Theoretische Natuurkunde, Rijksuniversiteit, Groningen, The Netherlands \\ R. V. Kadison \\ Department of Mathematics, University of Pennsylvania, Philadelphia, Pennsylvania, USA
}

Received September 25, 1974

\begin{abstract}
We study automorphisms of the CAR algebra which map the family of gauge-invariant, quasi-free states of the CAR algebra onto itself and show (Theorem 3.1) that they are one-particle automorphisms.
\end{abstract}

\section{Introduction}

The problem discussed in this paper arose from questions regarding equilibrium states of thermodynamical systems. Equilibrium states have been extensively discussed in the framework of $C^{*}$-algebras of observables (see, for example $[6,15])$. Such states are labeled by a very small number of parameters. For example, in the case of a gas of identical particles, the equilibrium states are labeled by the temperature, the chemical potential, and the average velocity of the particles - quantities which relate directly with the conserved quantities - energy, particle number, and total momentum. Since conserved quantities are in one-one correspondence with one-parameter groups of transformations which leave the Hamiltonian invariant, we can describe the situation in a way which remains meaningful for infinite systems. Equilibrium states of thermodynamical systems are labeled by a very small number of parameters which relate directly with oneparameter, automorphism groups of the observable algebra that commute with the time-evolution automorphisms. The fact that there are so few parameters involved, which is related to the fact that there are only a small number of one-parameter groups of automorphisms that commute with the time-evolution, is an aspect of the ergodic nature of most large physical systems. A proof based on the dynamics of the system is still lacking, even though Sinai has obtained very interesting results in this direction for a classical system of $N$ hard spheres.

Systems of particles without interaction do not behave ergodically in the above sense. This does not mean, however, that systems of noninteracting particles are uninteresting from the point of view of ergodicity. In $[7,8]$, the asymptotic time behavior of the free Fermi gas is discussed. It is found in [7] that, for increasing time, primary 'states of the CAR algebra are asymptotic to gauge-invariant, quasi-free states, provided these states satisfy a certain clustering property. In particular, primary, stationary (i.e. time-invariant) states with that clustering property are quasi-free. Quasi-free states $[1-5,9-14,16,17]$ are particularly simple states in the sense that they lack all except two-point correlations. Some 
basic facts about gauge-invariant, quasi-free states are given in Section 2 . These states are in one-one correspondence with positive operators in the unit ball, the so-called one-particle operators, acting on the one-particle Hilbert space $\mathscr{H}$. Stationary, quasi-free states of the free fermions are not labeled by a finite number of parameters, as in the interacting case, but by one-particle operators that commute with the Hamiltonian

$$
H\left(=-\left(\hbar^{2} / 2 m\right)\left(\partial^{2} / \partial x^{2}+\partial^{2} / \partial y^{2}+\partial^{2} / \partial z^{2}\right)=-\left(\hbar^{2} / 2 m\right) \Delta\right) .
$$

In particular, all operators of the form, $f(\hbar \partial / i \partial x, \hbar \partial / i \partial y, \hbar \partial / i \partial z)$ with $f$ positive and having essential supremum 1, define translationally-invariant, stationary states of the free fermion system. In this case, $f\left(p_{1}, p_{2}, p_{3}\right)$ is the momentum distribution of the particles.

The free fermion system is of particular interest to us because, as noted before, the set of all primary states that satisfy a certain clustering property is known. It is a subset of the gauge-invariant, quasi-free states. This result is an important tool in determining the automorphisms that commute with the free-time evolution. Since a clustering property that is somewhat stronger than that which holds for all primary states may well be a condition one must impose upon a physically meaningful state, we shall restrict our considerations to those automorphisms of the CAR algebra whose transposes preserve this property. Let $\alpha$ be such an automorphism that, in addition, commutes with the free-time evolution. Its transpose maps the set of stationary, quasi-free states, that satisfy the clustering property, onto itself. What can one conclude about $\alpha$ ?

Before attempting to solve this problem, one is faced with a more primitive question. What can be said about an automorphism $\alpha$ of the CAR algebra whose transpose maps the set of quasi-free states (or a certain subset of it) onto itself? Our main result (Theorem 3.1) states that, when the transpose leaves the set of gauge-invariant, quasi-free states stable, then, either the Fock state is mapped onto itself and there is a unitary operator $U$ on $\mathscr{H}$, such that $\alpha(a(f))=a(U f)$, or the Fock state is mapped onto the anti-Fock state and there is a conjugateunitary operator $W$ on $\mathscr{H}$, such that $\alpha(a(f))=a(W f)^{*}$, where $a(f)$ is the annihilation operator on Fock space.

A related result is stated in Theorem 4.1. A unitary operator on one-particle space defines, in an obvious manner, a unitary operator on $n$-particle space. Theorem 4.1 characterizes such unitary operators on $n$-particle space as those which map anti-symmetrized products of one-particle wave functions (product vectors) onto product vectors.

Section 2 is devoted to notation and a number of preliminary results, which are used throughout the paper. The main theorem is proven in Section 3; and Section 4 contains some related results.

\section{Some Preliminaries}

An infinite system of identical Fermi particles can be represented, insofar as their algebraic interrelations are concerned, by a $C^{*}$-algebra, $\mathfrak{A}$, the so-called CAR algebra. The abstract algebra $\mathfrak{A}$ may be characterized as the norm closure (completion) of an algebra generated by a countably-infinite family of pairwise-commuting, 
self-adjoint algebras each isomorphic to the algebra of $2 \times 2$ complex matrices and each containing the unit, $I$, of $\mathfrak{A}$. Each $*$-representation of $\mathfrak{A}$ gives rise to a representation of the canonical anticommutation relations (CAR), and conversely.

For our purposes, it is more useful to describe $\mathfrak{A}$ in its Fock representation. With $\mathscr{H}$ a complex Hilbert space and $\mathscr{H}_{n}$ the $n$-fold tensor product, so that, for $x_{1}, \ldots, x_{n}, y_{1}, \ldots, y_{n}$ in $\mathscr{H},\left\langle x_{1} \otimes \cdots \otimes x_{n} \mid y_{1} \otimes \cdots \otimes y_{n}\right\rangle=\left\langle x_{1} \mid y_{1}\right\rangle \cdots\left\langle x_{n} \mid y_{n}\right\rangle$, let $S_{n}^{-}$be the projection operator on $\mathscr{H}_{n}$ which assigns $\frac{1}{n !} \sum_{\sigma} \chi(\sigma) x_{\sigma(1)} \otimes \cdots \otimes x_{\sigma(n)}$ to $x_{1} \otimes \cdots \otimes x_{n}$, where $\sigma$ is a permutation of $\{1, \ldots, n\}$ and $\chi(\sigma)$ is +1 if $\sigma$ is even, -1 if $\sigma$ is odd. The range of $S_{n}^{-}$is the space $\mathscr{H}_{n}^{(a)}$ of antisymmetric tensors. We write $x_{1} \wedge \cdots \wedge x_{n}$ for $(n !)^{1 / 2} S_{n}^{-}\left(x_{1} \otimes \cdots \otimes x_{n}\right)$ (the "antisymmetrized, $n$-particle state with wave functions $\left.x_{1}, \ldots, x_{n}^{\prime \prime}\right)$. We have:

$$
\begin{aligned}
\left\langle x_{1} \wedge \cdots \wedge x_{n} \mid y_{1} \wedge \cdots \wedge y_{n}\right\rangle & =n !\left\langle x_{1} \otimes \cdots \otimes x_{n} \mid S_{n}^{-}\left(y_{1} \otimes \cdots \otimes y_{n}\right)\right\rangle \\
& =\sum_{\sigma} \chi(\sigma)\left\langle x_{1} \mid y_{\sigma(1)}\right\rangle \cdots\left\langle x_{n} \mid y_{\sigma(n)}\right\rangle=\operatorname{det}\left(\left\langle x_{i} \mid y_{j}\right\rangle\right) .
\end{aligned}
$$

Thus, assuming $x_{1} \wedge \cdots \wedge x_{n}$ and $y_{1} \wedge \cdots \wedge y_{n}$ are not 0 , they are orthogonal if and only if there are scalars $c_{1}, \ldots, c_{n}$, not all 0 , such that

$$
0=\sum_{i=1}^{n}\left\langle c_{i} x_{i} \mid y_{j}\right\rangle=\left\langle\sum_{i=1}^{n} c_{i} x_{i} \mid y_{j}\right\rangle ;
$$

that is, if and only if the space, $\left[x_{1}, \ldots, x_{n}\right]$, generated by $x_{1}, \ldots, x_{n}$, contains a non-zero vector $\left(\Sigma c_{i} x_{i}\right)$ orthogonal to $\left[y_{1}, \ldots, y_{n}\right]$. If, in addition, the intersection, $\left[x_{1}, \ldots, x_{n}\right] \cap\left[y_{1}, \ldots, y_{n}\right]$, of the spaces $\left[x_{1}, \ldots, x_{n}\right]$ and $\left[y_{1}, \ldots, y_{n}\right]$ has dimension $n-1$ (in this case, we say that the spaces are "perpendicular"), the projections with ranges $\left[x_{1}, \ldots, x_{n}\right]$ and $\left[y_{1}, \ldots, y_{n}\right]$ commute. It follows that $\left\{e_{i_{1}} \wedge \cdots \wedge e_{i_{n}}\right\}$ is an orthonormal basis for $\mathscr{H}_{n}^{(a)}$ if $\left\{e_{i}\right\}$ is an orthonormal basis for $\mathscr{H}$. Moreover, $x_{1} \wedge \cdots \wedge x_{n}=0$ if and only if $x_{1}, \ldots, x_{n}$ are linearly dependent (if and only if $\left[x_{1}, \ldots, x_{n}\right]$ has dimension less than $\left.n\right)$. Thus $z \in\left[x_{1}, \ldots, x_{n}\right]$, if $z \wedge x_{1} \wedge \cdots \wedge x_{n}=0$ and $x_{1} \wedge \cdots \wedge x_{n} \neq 0$. From this, if $x_{1} \wedge \cdots \wedge x_{n}=y_{1} \wedge \cdots \wedge y_{n} \neq 0$, then $\left[x_{1}, \ldots, x_{n}\right]$ $=\left[y_{1}, \ldots, y_{n}\right]$. On the other hand, if $\left[x_{1}, \ldots, x_{n}\right]=\left[y_{1}, \ldots, y_{n}\right]$, then, expressing each $y_{j}$ as a linear combination of $x_{1}, \ldots, x_{n}$, we see that $x_{1} \wedge \cdots \wedge x_{n}$ and $y_{1} \wedge \cdots \wedge y_{n}$ are scalar multiples of one another. We say that $x_{1} \wedge \cdots \wedge x_{n}$ is a product vector the exterior (or, wedge) product of $x_{1}, \ldots, x_{n}$.

The antisymmetric Fock space, $\mathscr{H}_{\mathscr{F}}^{(a)}$, is $\sum_{n=0}^{\infty} \oplus \mathscr{H}_{n}^{(a)}$. By definition $\mathscr{H}_{0}^{(a)}$ consists of complex scalar multiples of a single (unit) vector $\Phi_{0}$, the Fock vacuum; and $\mathscr{H}_{1}^{(a)}$ is $\mathscr{H}$. If $\mathscr{H}$ were finite dimensional, $\mathscr{H}_{\mathscr{F}}^{(a)}$ would be the (finite-dimensional) "exterior" algebra over $\mathscr{H}$. The mapping, $\wedge$, from the $n$-fold Cartesian product $\mathscr{H} \times \cdots \times \mathscr{H}$ to $\mathscr{H}_{n}^{(a)}$ which assigns $x_{1} \wedge \ldots \wedge x_{n}$ to $\left(x_{1}, \ldots, x_{n}\right)$ is an alternating, $n$-linear mapping. If $\tilde{a}$ is such a mapping of $\mathscr{H} \times \cdots \times \mathscr{H}$ into a space $\mathscr{K}$, there is a mapping $\hat{a}$ of $\mathscr{H}_{n}^{(a)}$ into $\mathscr{K}$ such that $\tilde{a}=\hat{a} \circ \wedge$. In particular if $T$ is a linear mapping of $\mathscr{H}$ into $\mathscr{K}$ then $\left(x_{1}, \ldots, x_{n}\right) \rightarrow T x_{1} \wedge \cdots \wedge T x_{n}$ is an alternating $n$-linear mapping of $\mathscr{H} \times \cdots \times \mathscr{H}_{\text {into }} \mathscr{K}_{n}^{(a)}$; so that there is a linear mapping $\hat{T}$ of $\mathscr{H}_{\mathscr{F}}^{(a)}$ into $\mathscr{K}_{\mathscr{F}}^{(a)}$ such that $\hat{T}\left(x_{1} \wedge \cdots \wedge x_{n}\right)=T x_{1} \wedge \cdots \wedge T x_{n}$. If $T$ is a unitary transformation of $\mathscr{H}$ onto $\mathscr{K}$, metric considerations apply and $\hat{T}$ is a unitary transformation of $\mathscr{H}_{\mathscr{F}}^{(a)}$ onto $\mathscr{K}_{\mathscr{F}}^{(a)}$. 
If $\|T\| \leqq 1, \tilde{\mathscr{H}}=\mathscr{H} \oplus \mathscr{H}, \dot{\mathscr{K}}=\mathscr{K} \oplus \mathscr{K}, P(x, y)=(x, 0)$, and $Q(u, v)=(u, 0)$, with $x, y$ in $\mathscr{H}$ and $u, v$ in $\mathscr{K}$, then there is a unitary transformation $U$ of $\mathscr{H}$ onto $\mathscr{\mathscr { K }}$ such that $Q U P(x, y)=(T x, 0)$ for all $x, y$ in $\mathscr{H}$. Then $\hat{U}$ is a unitary transformation of $\tilde{\mathscr{H}}_{n}^{(a)}$ onto $\tilde{\mathscr{K}}_{n}^{(a)}$ and $\hat{Q}$ is a projection of $\tilde{\mathscr{K}}_{n}^{(a)}$ onto $\mathscr{K}_{n}^{(a)}$. Since $\hat{T}$ is the restriction of $\hat{Q} \hat{U}$ to $\mathscr{H}_{n}^{(a)}$, we see that $\|\hat{T}\| \leqq 1$. If $T$ is a positive operator with pure point spectrum, computing norms with a basis of eigenvectors for $T$, we find that $\left\|\hat{T} \mid \mathscr{H}_{n}^{(a)}\right\|=\lambda_{1} \cdots \lambda_{n}$, where $\lambda_{1}, \ldots, \lambda_{n}$ are the $n$ largest eigenvalues of $T$ (multiplicity included). An approximation argument provides the corresponding result for a general positive operator; and polar decomposition provides a norm formula for a general bounded operator. A simple check yields $(\hat{T})^{*}=\hat{T}^{*}$.

Since $\left(f_{1}, \ldots, f_{n}\right) \rightarrow f \wedge f_{1} \wedge \ldots \wedge f_{n}$ is an alternating, $n$-linear mapping, there is a linear mapping, $a_{n}(f)^{*}$, of $\mathscr{H}_{n}^{(a)}$ into $\mathscr{H}_{n+1}^{(a)}$ with value $f \wedge f_{1} \wedge \cdots \wedge f_{n}$ at $f_{1} \wedge \cdots \wedge f_{n}$. The family $\left\{a_{n}(f)^{*}\right\}$ defines a mapping $a(f)^{*}$ on $\mathscr{H}_{\mathscr{F}}^{(a)}$. With $\left\{e_{i}\right\}$ an orthonormal basis for $\mathscr{H}, a\left(e_{1}\right)^{*}$ maps $\left\{e_{i_{1}} \wedge \cdots \wedge e_{i_{n}}: 1 \notin\left\{i_{1}, \ldots, i_{n}\right\}, n=0,1,2, \ldots\right\}$ onto an orthonormal basis for the orthogonal complement, $\mathscr{H}_{\mathscr{F}}^{(a)} \ominus \mathscr{K}$; and $a\left(e_{1}\right)^{*}$ annihilates this complement. Thus $a\left(e_{1}\right)^{*}$ is a partial isometry with initial space $\mathscr{K}$ and final space $\mathscr{H}_{\mathscr{F}}^{(a)} \ominus \mathscr{K}$. It follows that $I=a\left(e_{1}\right)^{*} a\left(e_{1}\right)+a\left(e_{1}\right) a\left(e_{1}\right)^{*}$ $\left(=\left\{a\left(e_{1}\right), a\left(e_{1}\right)^{*}\right\}_{+}\right)$. More generally $a(f) a(f)^{*}+a(f)^{*} a(f)=\langle f \mid f\rangle I$. Polarization of this yields: $\left\{a(f), a(g)^{*}\right\}_{+}=\langle f \mid g\rangle I$. We note that our inner product, $\langle f \mid g\rangle$, is linear in $g$ and conjugate linear in $f$. We have $\left\{a(f)^{*}, a(g)^{*}\right\}_{+}=0$, as well. A conjugate-linear mapping $f \rightarrow a(f)$ of $\mathscr{H}$ onto operators $a(f)$ on a Hilbert space satisfying the relations (canonical anticommutation relations)

$$
\left\{a(f), a(g)^{*}\right\}_{+}=\langle f \mid g\rangle I,\{a(f), a(g)\}_{+}=0
$$

is said to be a representation of the CAR. The particular representation we have exhibited on $\mathscr{H}_{\mathscr{F}}^{(a)}$ is called the Fock representation.

We can exhibit the annihilator $a(f)$ as explicitly as we described the creator $a(f)^{*}$ by expanding the determinant expression for $\left\langle f \wedge y_{2} \wedge \cdots \wedge y_{n} \mid x_{1} \wedge \cdots \wedge x_{n}\right\rangle$ in terms of its first row:

$$
\begin{aligned}
& \left\langle f \wedge y_{2} \wedge \cdots \wedge y_{n} \mid x_{1} \wedge \cdots \wedge x_{n}\right\rangle \\
& \quad=\left\langle y_{2} \wedge \cdots \wedge y_{n} \mid a(f)\left(x_{1} \wedge \cdots \wedge x_{n}\right)\right\rangle \\
& \quad=\sum_{j=1}^{n}(-1)^{j+1}\left\langle f \mid x_{j}\right\rangle\left\langle y_{2} \wedge \cdots \wedge y_{n} \mid x_{1} \wedge \cdots \wedge x_{j-1} \wedge x_{j+1} \wedge \cdots \wedge x_{n}\right\rangle
\end{aligned}
$$

so that

$$
a(f)\left(x_{1} \wedge \cdots \wedge x_{n}\right)=\sum_{j=1}^{n}(-1)^{j+1}\left\langle f \mid x_{j}\right\rangle x_{1} \wedge \cdots \wedge x_{j-1} \wedge x_{j+1} \wedge \cdots \wedge x_{n} .
$$

With $E$ a projection on $\mathscr{H}$, we denote by $\mathfrak{A}_{0}(E)$ and $\mathfrak{U}(E)$ the $*$-algebra and $C^{*}$-algebra, respectively, on $\mathscr{H}_{\mathscr{F}}^{(a)}$ generated by $\{a(f): E f=f\}$. We write $\mathfrak{A}_{0}$ and $\mathfrak{A}$ in place of $\mathfrak{U}_{0}(I)$ and $\mathfrak{U}(I)$. The $C^{*}$-algebra $\mathfrak{A}$ is the CAR algebra and its action on $\mathscr{H}_{\mathscr{F}}^{(a)}$ is called its Fock representation. The state $\phi_{0}$ of $\mathfrak{A}$ for which $\phi_{0}(A)=\left\langle\Phi_{0} \mid A \Phi_{0}\right\rangle$ is called the Fock (vacuum) state of $\mathfrak{A}$. Note that each $a(f)$ is in its left kernel $\mathscr{K}\left(\phi_{0}\left(a(f)^{*} a(f)\right)=0\right)$; so that each product of annihilators and creators (monomial) in which an annihilator appears to the right is in $\mathscr{K}$. Now each monomial is a sum of monomials in which all creators are to the left of all annihilators (we say that such a monomial is Wick-ordered - and anti-Wick-ordered if all creators are to the right of all annihilators); so that $\phi_{0}$ annihilates all Wick-ordered monomials 
in $\mathfrak{U}_{0}$ other than $I$. These monomials span the null space of $\phi_{0}$ on $\mathfrak{A}_{0}$. If $\varrho$ is a state of $\mathfrak{U}$ and $\varrho \leqq 2 \phi_{0}$, then $a(f)$ is in the left kernel of $\varrho$. Thus $\varrho$ and $\phi_{0}$ have the same null space in $\mathfrak{U}_{0}$ and agree at $I$. Hence $\varrho=\phi_{0}$; and $\phi_{0}$ is a pure state of $\mathfrak{A}$. Exactly the same considerations apply to the restriction of $\phi_{0}$ to $\mathfrak{U}(E)$, for each projection $E$ on $\mathscr{H}$. Thus the restriction of $\phi_{0}$ to $\mathfrak{A}(E)$ is pure.

The Hilbert space $\overline{\mathscr{H}}$, obtained from $\mathscr{H}$ by assigning an element $\bar{f}$ to each $f$ in $\mathscr{H}$, defining $(\overline{\bar{c} f+g})$ to be $c \bar{f}+\bar{g}$ and $\langle\bar{f} \mid \bar{g}\rangle$ to be $\langle g \mid f\rangle$, produces $\overline{\mathscr{H}}_{\mathscr{F}}^{(a)}$, anti-Fock space, and $\bar{\phi}_{0}$ is the anti-Fock vacuum. The mapping $f \rightarrow a(\bar{f})^{*}(=\bar{a}(f))$ is a representation of the CAR (over $\mathscr{H}$ ), the anti-Fock representation; and the mapping $a(f) \rightarrow \bar{a}(f)$ extends to a $*$-isomorphism, $A \rightarrow \bar{A}$, of the CAR algebra $\mathfrak{U}$ over $\mathscr{H}$ onto the CAR algebra $\overline{\mathfrak{U}}$ over $\overline{\mathscr{H}}$. The state $\phi_{I}$ of $\mathfrak{U}$ defined by $A \rightarrow\left\langle\bar{\Phi}_{0} \mid \bar{A} \bar{\Phi}_{0}\right\rangle$ is the anti-Fock state of $\mathfrak{A}$. Each $a(f)^{*}$ is in the left kernel of $\phi_{I}$; so that, replacing $a(f)$ by $a(f)^{*}$ and using anti-Wick-ordered monomials instead of Wick-ordered monomials in the argument above, we have that the restriction of $\phi_{I}$ to each $\mathfrak{U}(E)$ is pure.

Since $\phi_{0}$ is pure and $\Phi_{0}$ is cyclic for $\mathfrak{A}$, the weak-operator closure, $\mathfrak{A}^{-}$, of $\mathfrak{A}$, is $\mathscr{B}\left(\mathscr{H}_{\mathscr{F}}^{(a)}\right)$, the algebra of all bounded operators on $\mathscr{H}_{\mathscr{F}}^{(a)}$. Similarly $\mathfrak{A}(E)^{-} \tilde{E}_{0}$ $=\mathscr{B}\left(\left[\mathfrak{U}(E) \Phi_{0}\right]\right)$, where $\hat{E}_{0}$ is the projection (in $\left.\mathfrak{U}(E)^{\prime}\right)$ with range $\left[\mathfrak{U}(E) \Phi_{0}\right]$. If $U_{E}$ is $(\overline{I-2 E})$, then $U_{E} \Phi_{0}=\Phi_{0}, a(g) U_{E}=U_{E} a(g)$, for each $g$ in $(I-E)(\mathscr{H})$, and $a(f) U_{E}=-U_{E} a(f)$, for each $f$ in $E(\mathscr{H})$. If $A_{0}$ is an even monomial in $\mathfrak{A}_{0}(I-E)$ (that is, $A_{0}$ is the product of an even total number of annihilators and creators) and $A_{1}$ is an odd monomial in $\mathfrak{U}_{0}(I-E)$, then $A_{0}$ and $A_{1} U_{E}$ lie in $\mathfrak{A}(E)^{\prime}$. Since $\mathfrak{U}_{0}(E)$ and $\mathfrak{U}_{0}(I-E)$ generate $\mathfrak{U}_{0}$ and $\Phi_{0}$ is cyclic for $\mathfrak{U}_{0}$;

$$
\mathscr{H}_{\mathscr{F}}^{(a)}=\left[\mathfrak{U}_{0} \Phi_{0}\right]=\left[\mathfrak{U}(E) \mathfrak{U}(I-E) \Phi_{0}\right]=\left[\mathfrak{U}(E) \mathfrak{U}(E)^{\prime} \Phi_{0}\right] .
$$

Thus $\tilde{E}_{0}$ has central carrier $I$ in $\mathfrak{U}(E)^{-}$; and the mapping $\imath_{E}$ of $\mathfrak{U}(E)^{-} \tilde{E}_{0}$ onto $\mathfrak{U}(E)^{-}$which assigns $A$ to $A \tilde{E}_{0}$ is a $*$-isomorphism.

Now, $a(f) \Phi_{0}=0$ and, when $E f=0, a(f)\left(\mathfrak{U}_{0}(E) \Phi_{0}\right)=(0)$. Thus $a(f) \tilde{E}_{0}=0$ and $\hat{E}_{0} a(f)^{*}=0$, when $E f=0$; so that $\tilde{E}_{0} A \tilde{E}_{0}=\lambda \tilde{E}_{0}$ when $A$ is in $\mathfrak{A}_{0}(I-E)$. It follows that $B \rightarrow \tilde{E}_{0} B \tilde{E}_{0}$ is a (completely-) positive, linear mapping of $\mathscr{B}\left(\mathscr{H}_{\mathscr{F}}^{(a)}\right)$ onto $\mathfrak{A}(E)^{-} \tilde{E}_{0}$. The composition of this mapping with $\imath_{E}$ is a completely-positive, linear mapping, $\psi_{E}$, of $\mathscr{B}\left(\mathscr{H}_{\mathscr{F}}^{(a)}\right)$ onto $\mathfrak{A}(E)^{-}$. By construction of $\psi_{E}$,

$$
\psi_{E}\left(a\left(x_{n}\right) * \ldots a\left(x_{1}\right) * a\left(y_{1}\right) \ldots a\left(y_{m}\right)\right)=a\left(E x_{n}\right)^{*} \ldots a\left(E x_{1}\right)^{*} a\left(E y_{1}\right) \ldots a\left(E y_{m}\right) .
$$

More generally:

Proposition 2.1. If $T$ is a linear transformation of one Hilbert space, $\mathscr{H}$, into another, $\mathscr{K}$, and $\|T\| \leqq 1$, then the mapping

$$
a\left(x_{n}\right)^{*} \ldots a\left(x_{1}\right)^{*} a\left(y_{1}\right) \ldots a\left(y_{m}\right) \rightarrow a\left(T x_{n}\right)^{*} \ldots a\left(T x_{1}\right)^{*} a\left(T y_{1}\right) \ldots a\left(T y_{m}\right)
$$

extends (uniquely) to a completely-positive, linear mapping $\psi_{T}$ of the CAR algebra, $\mathfrak{U}_{\mathscr{H}}$, over $\mathscr{H}$ into the CAR algebra, $\mathfrak{U}_{\mathscr{K}}$, over $\mathscr{K}$.

Proof. If $\tilde{\mathscr{H}}=\mathscr{H} \oplus \mathscr{H}, \tilde{\mathscr{K}}=\mathscr{K} \oplus \mathscr{K}, P\left(h, h^{\prime}\right)=(h, 0)$ for $h, h^{\prime}$ in $\mathscr{H}, Q\left(k, k^{\prime}\right)$ $=(k, 0)$ for $k, k^{\prime}$ in $\mathscr{K}$, and $\tilde{T}\left(h, h^{\prime}\right)=(T h, 0)$, then there is a unitary transformation $U$ of $\tilde{\mathscr{H}}$ onto $\tilde{\mathscr{K}}$ such that $Q U P=\tilde{T}$. The mapping $a(f) \rightarrow a(U f)$ extends, uniquely, to a $*$-isomorphism of $\mathfrak{U}_{\tilde{\mathscr{H}}}$ onto $\mathfrak{U}_{\tilde{\mathscr{K}}}$. The composition of the restriction of this isomorphism to $\mathfrak{I}_{\mathscr{H}}(P)$ and $\psi_{Q}$ is $\psi_{T}$. 
We note that the characterization of $\psi_{T}$ as the result of distributing $T$ throughout a Wick-ordered monomial is independent of the ordering only if $T$ is an isometry; for $\psi_{T}\left(a(f) a(f)^{*}\right)=\psi_{T}\left(I-a(f)^{*} a(f)\right)=I-a(T f)^{*} a(T f) \neq a(T f) a(T f)^{*}$, when $\|f\|=1$, unless $\langle T f \mid T f\rangle=1$.

If $A \in \mathscr{B}(\mathscr{H})$ and $0 \leqq A \leqq I$, we call $\phi_{I^{\circ}} \psi_{A^{\frac{1}{2}}}$ the gauge-invariant, quasi-free state of $\mathfrak{U}$ with one-particle operator $A$. We write $\phi_{A}$ for this state and note that there is no conflict between this notation and the designation of the Fock and anti-Fock states of by $\phi_{0}$ and $\phi_{I}$ (i.e. these states are quasi-free with one-particle operators 0 and $I$, respectively). Note that

$$
\begin{aligned}
& \phi_{A}\left(a\left(f_{n}\right)^{*} \ldots a\left(f_{1}\right)^{*} a\left(g_{1}\right) \ldots a\left(g_{n}\right)\right) \\
&=\phi_{I}\left(a\left(A^{\frac{1}{2}} f_{n}\right)^{*} \ldots a\left(A^{\frac{1}{2}} f_{1}\right)^{*} a\left(A^{\frac{1}{2}} g_{1}\right) \ldots a\left(A^{\frac{1}{2}} g_{n}\right)\right) \\
&=\left\langle\bar{\Phi}_{0} \mid a\left(\overline{A^{\frac{1}{2}} f_{n}}\right) \ldots a\left(\overline{A^{\frac{1}{2}} f_{1}}\right) a\left(\overline{A^{\frac{1}{2}} g_{1}}\right)^{*} \ldots a\left(\overline{A^{\frac{1}{2}} g_{n}}\right)^{*} \bar{\Phi}_{0}\right\rangle \\
&=\left\langle\overline{A^{\frac{1}{2}} f_{1}} \wedge \ldots \wedge \overline{A^{\frac{1}{2}} f_{n}} \mid \overline{A^{\frac{1}{2}} g_{1}} \wedge \ldots \wedge \overline{A^{\frac{1}{2}} g_{n}}\right\rangle \\
&=\operatorname{det}\left(\left\langle\overline{A^{\frac{1}{2}} f_{i}} \mid \overline{A^{\frac{1}{2}} g_{j}}\right\rangle\right)=\operatorname{det}\left(\left\langle g_{j} \mid A f_{i}\right\rangle\right) \\
&=\operatorname{det}\left(\left\langle g_{i} \mid A f_{j}\right\rangle\right)=\left\langle g_{1} \wedge \ldots \wedge g_{n} \mid A f_{1} \wedge \ldots \wedge A f_{n}\right\rangle .
\end{aligned}
$$

Proposition 2.2. If $E$ is a finite-dimensional projection on $\mathscr{H}$ with $\left\{e_{1}, \ldots, e_{n}\right\}$ an orthonormal basis for $E(\mathscr{H})$, then $\phi_{E}(T)=\left\langle e_{1} \wedge \cdots \wedge e_{n} \mid T\left(e_{1} \wedge \cdots \wedge e_{n}\right)\right\rangle$.

Proof. Let $\left\{e_{j}\right\}$ be an orthonormal basis for $\mathscr{H}$, and $T$ be a Wick-ordered monomial in annihilators and creators corresponding to basis elements. Then $\left\langle e_{1} \wedge \cdots \wedge e_{n} \mid T\left(e_{1} \wedge \cdots \wedge e_{n}\right)\right\rangle$ is 0 unless $T$ has the form $a\left(e_{i_{\sigma(m)}}\right)^{*} \ldots a\left(e_{i_{\sigma(1)}}\right)^{*}$ .$a\left(e_{i_{1}}\right) \ldots a\left(e_{i_{m}}\right)$, with $\left\{i_{1} \ldots, i_{m}\right\}$ an $m$-element subset of $\{1, \ldots, n\}$, in which case its value and that of $\phi_{E}(T)$ is $\chi(\sigma)$. If $T$ does not have this form $\psi_{E}(T)=0$, so $\phi_{E}(T)=0$. Thus our equality holds.

If follows that $\phi_{E}$ is pure when $E$ is a finite-dimensional projection on $\mathscr{H}$. More generally, if $E$ is any orthogonal projection on $\mathscr{H}$ and $\varrho$ is a state of $\mathfrak{A}$ such that $\varrho \leqq 2 \phi_{E}$ then the restrictions of $\varrho$ to $\mathfrak{U}(E)$ and $\mathfrak{U}(I-E)$ coincide with those of $\phi_{I}$ and $\phi_{0}$, respectively. Using the fact that monomials $A$ and $A^{\prime}$ in $\mathfrak{U}_{0}(E)$ and $\mathfrak{A}_{0}(I-E)$, respectively, commute or anti-commute and that Wick-ordered monomials are in the left or right kernels of $\phi_{0}$ while anti-Wick ordered monomials are in the left or right kernels of $\phi_{I}$, (other than $c I, c \neq 0$ ), we conclude that $\varrho\left(A A^{\prime}\right.$ ) $=\varrho(A) \varrho\left(A^{\prime}\right)$. The same is true for $A$ in $\mathfrak{U}(E)$ and $A^{\prime}$ in $\mathfrak{A}(I-E)$. Thus $\varrho=\phi_{E}$ and $\phi_{E}$ is pure.

If $0 \leqq A_{0} \leqq I$ with $A_{0}\left(\neq A_{0}^{2}\right)$ in $\mathscr{B}(\mathscr{H})$, using the Spectral Theorem, there is a one-dimensional projection $E_{1}$ on $\mathscr{H}$ and a positive number $t$ such that $0 \leqq A_{1} \leqq I$ and $0 \leqq A_{2} \leqq I$, where $A_{1}=A_{0}+t E_{1}$ and $A_{2}=A_{0}-t E_{1}$. Computing with an orthonormal basis $\left\{e_{j}\right\}$ for $\mathscr{H}$ such that $E_{1} e_{1}=e_{1}$, we have that $\phi_{A_{0}}=\frac{1}{2}\left(\phi_{A_{1}}+\phi_{A_{2}}\right)$. To see this, note that

$$
\begin{aligned}
\phi_{A_{k}}\left(a\left(e_{i_{n}}\right)^{*} \ldots a\left(e_{i_{1}}\right)^{*} a\left(e_{j_{1}}\right) \ldots a\left(e_{j_{n}}\right)\right) \\
=\left\langle e_{j_{1}} \wedge \ldots \wedge e_{j_{n}} \mid A_{k} e_{i_{1}} \wedge \ldots \wedge A_{k} e_{i_{n}}\right\rangle,
\end{aligned}
$$

where $k=0,1,2$; and that $A_{0} e_{j}=A_{1} e_{j}=A_{2} e_{j}$, when $j \neq 1$. Thus $\phi_{A}$ is pure if and only if $A$ is a projection.

From the foregoing, if $E$ is a finite-dimensional projection, $\phi_{E}$ is a pure, gaugeinvariant, quasi-free state equivalent to the Fock state. Conversely, if $E$ is a projec- 
tion on one-particle space $\mathscr{H}$ and $\phi_{E}$ is equivalent to the Fock state, then $E$ is a finite-dimensional projection. This follows as a special case of [12; Theorem 2.8]. A direct proof is not difficult. If $\phi_{E}=\omega_{x} \mid \mathfrak{A}$, for some unit vector $x$ of $\mathscr{H}_{\mathscr{F}}^{(a)}$, then $1=\phi_{E}\left(a\left(e_{j}\right)^{*} a\left(e_{j}\right)\right)=\omega_{x}\left(a\left(e_{j}\right)^{*} a\left(e_{j}\right)\right)$, where $\left\{e_{j}\right\}$ is an orthonormal basis for $E(\mathscr{H})$. Thus $a\left(e_{j}\right) * x=0$, for each $j$. If

$$
x=\sum_{i_{1}<\cdots<i_{n} ; j_{1}<\cdots<j_{m}} c_{i_{1} \ldots i_{n} ; j_{1} \ldots j_{m}} e_{i_{1}} \wedge \cdots \wedge e_{i_{n}} \wedge e_{j_{1}}^{\prime} \wedge \cdots \wedge e_{j_{m}}^{\prime},
$$

where $\left\{e_{j}^{\prime}\right\}$ is an orthonormal basis for $(I-E)(\mathscr{H})$, then

$$
0=a\left(e_{j}\right)^{*} x=\Sigma c_{i_{1} \ldots i_{n} ; j_{1} \ldots j_{m}} e_{j} \wedge e_{i_{1}} \wedge \cdots \wedge e_{i_{n}} \wedge e_{j_{1}}^{\prime} \wedge \cdots \wedge e_{j_{m}}^{\prime}
$$

so that $j \in\left\{i_{1}, \ldots, i_{n}\right\}$ unless $c_{i_{1} \ldots i_{n} ; j_{1} \ldots j_{m}}=0$. If $E(\mathscr{H})$ is infinite-dimensional, we can choose $j$ not in $\left\{i_{1}, \ldots, i_{n}\right\}$; and $x=0$, contradicting the assumption that $x$ is a unit vector. Thus $E$ is a finite-dimensional projection.

\section{Automorphisms Preserving Gauge-Invariant, Quasi-Free States}

Our main result is contained in the following theorem.

Theorem 3.1. If $\mathfrak{U}$ is the CAR algebra in its Fock representation on the complex Hilbert space $\mathscr{H}_{\mathscr{F}}^{(a)}$ of antisymmetric tensors over $\mathscr{H}$ and $\alpha$ is an automorphism of $\mathfrak{U}$ whose transpose $\hat{\alpha}$ carries the set of gauge-invariant, quasi-free states onto itself, then, either the Fock state is mapped onto itself by $\hat{\alpha}$ and there is a unitary operator $U$ on $\mathscr{H}$ such that $\alpha(a(f))=a(U f)$, or the Fock state is mapped onto the anti-Fock state by $\hat{\alpha}$ and there is a conjugate-linear, unitary operator $W$ on $\mathscr{H}$ such that $\alpha(a(f))=a(W f)^{*}$.

The proof of Theorem 3.1 will be effected with the aid of the following results (3.2-3.13). During its course, we will note, in the case where $\hat{\alpha}\left(\phi_{0}\right)=\phi_{0}$, that $\hat{U}$ implements $\alpha$ (see Section 2).

Lemma 3.2. The image of $\phi_{0}$ under $\hat{\alpha}$ is either $\phi_{0}$ or $\phi_{I}$.

Proof. Since $\hat{\alpha}\left(\phi_{0}\right)$ is pure and, by assumption, a quasi-free, gauge-invariant state of $\mathfrak{A} ; \hat{\alpha}\left(\phi_{0}\right)=\phi_{E_{0}}$, for some projection $E_{0}$ on $\mathscr{H}$ (see Section 2). If $\pi$ is the representation of $\mathfrak{U}$ on $\mathscr{H}_{\pi}$ determined by $\phi_{E_{0}}$, then the mapping $A \Phi_{0} \rightarrow \pi\left(\alpha^{-1}(A)\right) x_{E_{0}}$, where $x_{E_{0}}$ is a unit vector in $\mathscr{H}_{\pi}$ such that $\phi_{E_{0}}(A)$ $=\left\langle x_{E_{0}} \mid \pi(A) x_{E_{0}}\right\rangle$ for all $A$ in $\mathfrak{U}$, extends to a unitary transformation $U$ of $\mathscr{H}_{\mathscr{F}}^{(a)}$ onto $\mathscr{H}_{\pi}$. If $E_{0}$ is neither 0 nor $I$, there is a unit vector $f$ in $E_{0}(\mathscr{H})$ and a unit vector $g$ orthogonal to $E_{0}(\mathscr{H})$. We shall arrive at a contradiction from this assumption, so that $E_{0}$ is either 0 or $I$ and $\hat{\alpha}\left(\phi_{0}\right)$ is either the Fock or anti-Fock state.

If $E_{1}$ is the projection on $\mathscr{H}$ with $\left\{x:\langle x \mid f\rangle=0, E_{0} x=x\right\}$ as range then $\phi_{E_{0}}(A)=\phi_{E_{1}}\left(a(f) A a(f)^{*}\right)$, for $\phi_{E_{1}}\left(a(f) a(h) a(h)^{*} a(f)^{*}\right)=0$, when $E_{1} h=h$ or when $h=f$; and $\phi_{E_{1}}\left(a(f) a(k)^{*} a(k) a(f)^{*}\right)=\phi_{E_{1}}\left(a(f) a(f)^{*} a(k)^{*} a(k)\right)=0$, when $E_{0} k=0$. Thus $\phi_{E_{1}}$ is equivalent to $\phi_{E_{0}}$; and there is a vector $x_{E_{1}}$ in $\mathscr{H}_{\pi}$ such that $\phi_{E_{1}}(A)$ $=\left\langle x_{E_{1}} \mid \pi(A) x_{E_{1}}\right\rangle$. Similarly, if $E_{2}$ is the projection on $\mathscr{H}$ with range generated by $E_{1}(\mathscr{H})$ and $g$, then $\phi_{E_{2}}(A)=\phi_{E_{1}}\left(a(g) A a(g)^{*}\right)$, for all $A$ in $\mathfrak{A}$; and there is a vector $x_{E_{2}}$ in $\mathscr{H}_{\pi}$ such that $\phi_{E_{2}}(A)=\left\langle x_{E_{2}} \mid \pi(A) x_{E_{2}}\right\rangle=\left\langle\pi\left(a(g)^{*}\right) x_{E_{1}} \mid \pi(A) \pi\left(a(g)^{*}\right) x_{E_{1}}\right\rangle$. 
Since $\pi$ is irreducible, $x_{E_{2}}=c_{2} \pi\left(a(g)^{*}\right) x_{E_{1}}$, and, similarly, $x_{E_{0}}=c_{0} \pi\left(a(f)^{*}\right) x_{E_{1}}$, where $c_{0}$ and $c_{2}$ are scalars of modulus 1 . Now,

$$
2^{-\frac{1}{2}}\left(x_{E_{0}}+x_{E_{2}}\right)=\pi\left(a\left(2^{-\frac{1}{2}}\left(c_{0} f+c_{2} g\right)\right)^{*}\right) x_{E_{1}}\left(=x_{F}\right) ;
$$

so that $\phi_{F}(A)=\left\langle x_{F} \mid \pi(A) x_{F}\right\rangle$, for all $A$ in $\mathfrak{A}$, where $F$ is the projection on $\mathscr{H}$ with range generated by $E_{1}(\mathscr{H})$ and $c_{0} f+c_{2} g$. If $U x_{1}=x_{F}$ and $U x_{2}=x_{E_{2}}$ then, since $U \Phi_{0}=x_{E_{0}}, \Phi_{0}+x_{2}=2^{\frac{1}{2}} x_{1}$. Noting that $U A U^{-1}=\pi\left(\alpha^{-1}(A)\right)$, for each $A$ in $\mathfrak{A}$, we have $\left\langle x_{2} \mid A x_{2}\right\rangle=\left\langle x_{E_{2}} \mid U A U^{-1} x_{E_{2}}\right\rangle=\left\langle x_{E_{2}} \mid \pi\left(\alpha^{-1}(A)\right) x_{E_{2}}\right\rangle=\phi_{E_{2}}\left(\alpha^{-1}(A)\right)$. Similarly $\left\langle x_{1} \mid A x_{1}\right\rangle=\phi_{F}\left(\alpha^{-1}(A)\right)$ and $\left\langle\Phi_{0} \mid A \Phi_{0}\right\rangle=\phi_{E_{0}}\left(\alpha^{-1}(A)\right)$. Thus $\omega_{x_{1}} \mid \mathfrak{A}$ and $\omega_{x_{2}} \mid \mathfrak{X}$ are pure, gauge-invariant, quasi-free states which transform under $\hat{\alpha}$ into $\phi_{F}$ and $\phi_{E_{2}}$. Since $\phi_{F}$ and $\phi_{E_{2}}$ are equivalent to $\phi_{E_{0}}, \omega_{x_{1}} \mid \mathfrak{A}$ and $\omega_{x_{2}} \mid \mathfrak{A}$ are equivalent to $\phi_{0}$. From Proposition 2.2, $x_{1}$ and $x_{2}$ are product vectors in $\mathscr{H}_{\mathscr{F}}^{(a)}$. But $\Phi_{0}+x_{2}=2^{\frac{1}{2}} x_{1}$, and each product vector lies in an $n$-particle space. Thus $x_{1}$ and $x_{2}$ are multiples of $\Phi_{0}$; and $\phi_{E_{2}}=\phi_{0}$, contrary to the choice of $E_{2}$ different from 0 .

In case $\hat{\alpha}\left(\phi_{0}\right)=\phi_{I}, \hat{\alpha}^{\prime}\left(\phi_{0}\right)=\phi_{0}$, where $\alpha^{\prime}=\alpha \circ \sigma$ and $\sigma(a(f))=a\left(W_{0} f\right)^{*}$ with $W_{0}$ a conjugate-unitary operator on $\mathscr{H}$ (obtained, for example, by transforming each linear combination of elements in an orthonormal basis for $\mathscr{H}$ into the linear combination resulting from replacing each coefficient by its complex-conjugate). Since $\sigma$ determines an automorphism of $\mathfrak{A}$ which interchanges the Fock and antiFock states and which maps the set of gauge-invariant, quasi-free states onto itself; $\hat{\alpha}^{\prime}$ maps the set of gauge-invariant, quasi-free states onto itself. If we prove that there is a unitary operator $U_{0}$ on $\mathscr{H}$ such that $\alpha^{\prime}(a(f))=a\left(U_{0} f\right)$, then $\alpha\left(a\left(W_{0} f\right)^{*}\right)=a\left(U_{0} f\right)$, and $\alpha(a(f))=a\left(U_{0} W_{0}^{*} f\right)^{*}$, with $W$ the conjugate-unitary operator $U_{0} W_{0}^{*}$ on $\mathscr{H}$.

We assume, henceforth, that $\hat{\alpha}\left(\phi_{0}\right)=\phi_{0}$ and use the notation of Theorem 3.1 throughout the remainder of this section. With this assumption, $U$, constructed in Lemma 3.2 , is a unitary operator on $\mathscr{H}_{\mathscr{F}}^{(a)}$ which carries product vectors onto product vectors. Although the components of the argument proving that are to be found in the proof of Lemma 3.2, we make the statement and proof explicit in Lemma 3.3. Note that the automorphism $\sigma$, above, is a special case of the larger class of Bogoliubov transformations. In Section 2, we introduced the notation $\hat{T}$ to denote a certain transformation on $\mathscr{H}_{\mathscr{F}}^{(a)}$ arising from $T$ defined on $\mathscr{H}$. In Lemma 3.3 and the results following, we construct a unitary operator $\hat{U}$ on $\mathscr{H}_{\mathscr{F}}^{(a)}$. We will eventually locate a unitary operator $U$ on $\mathscr{H}$ for which $\hat{U}$ is the transformation on $\mathscr{H}_{\mathscr{F}}^{(a)}$ arising from it - justifying this notation.

Lemma 3.3. There is a unitary operator $\hat{U}$ on $\mathscr{H}_{\mathscr{F}}^{(a)}$ which implements $\alpha$ and maps product vectors onto product vectors.

Proof. Since $\hat{\alpha}\left(\phi_{0}\right)=\phi_{0}$, the mapping $A \Phi_{0} \rightarrow \alpha(A) \Phi_{0}$ extends to a unitary operator $\hat{U}$ on $\mathscr{H}_{\mathscr{F}}^{(a)}$ such that $\hat{U} A \hat{U}^{*}=\alpha(A)$. We show that $\hat{U}\left(x_{1} \wedge \cdots \wedge x_{n}\right)$ is a product vector, for all $x_{1}, \ldots, x_{n}$ in $\mathscr{H}$. Since $x_{1} \wedge \ldots \wedge x_{n}$ is a scalar multiple of the wedge-product of an orthonormal set of vectors (a basis for $\left[x_{1}, \ldots, x_{n}\right]$, when $x_{1} \wedge \cdots \wedge x_{n} \neq 0$ ), we may assume that $\left\{x_{1}, \ldots, x_{n}\right\}$ is an orthonormal set. If $E_{0}$ is the projection with range $\left[x_{1}, \ldots, x_{n}\right], \phi_{E_{0}}=\omega_{x_{1} \wedge \ldots \wedge x_{n}}$, from Proposition 2.2. As $\alpha$ is implemented by a unitary operator on $\mathscr{H}_{\mathscr{F}}^{(a)}, \hat{\alpha}\left(\phi_{E_{0}}\right)$ is a vector state of $\mathfrak{A}$. By assumption, $\hat{\alpha}\left(\phi_{E_{0}}\right)$ is a gauge-invariant quasi-free state of $\mathfrak{A}$ (equivalent to $\phi_{0}$, from the preceding remark). Thus $\hat{\alpha}\left(\phi_{E_{0}}\right)=\omega_{y_{1} \wedge \cdots \wedge y_{m}} \mid \mathfrak{A}$ where $y_{1} \wedge \cdots \wedge y_{m}$ $=\hat{U}^{*}\left(x_{1} \wedge \cdots \wedge x_{n}\right)$. 
A linear transformation (such as $\hat{U}$, above) which is defined on a subspace of $\mathscr{H}_{\mathscr{F}}^{(a)}$ and maps product vectors onto product vectors will be said to be a product linear transformation (or product unitary, etc.).

Lemma 3.4. The intersection of an infinite, commuting family of m-dimensional projections each pair of which has $m-1$ dimensional intersection is $m-1$ dimensional.

Proof. Since each projection is $m$-dimensional, each is the sum of $m$ onedimensional projections. Since the family is commutative, we can find an orthogonal set of one-dimensional projections such that each projection of our commuting family is the sum of $m$ of them. In this way, our problem reduces to showing that if $S=\bigcup_{a} S_{a}$ where $\left\{S_{a}\right\}$ is an infinite family of sets each of which has $m$ elements and such that each pair has intersection with $m-1$ elements, then $\bigcap_{a} S_{a}$ has $m-1$ elements (in other words, each $S_{c}$ contains $S_{a} \cap S_{b}$, when $a \neq b$ ). Suppose $S_{c}$ does not contain $S_{a} \cap S_{b}$. Then $S_{a} \cap S_{c}$ and $S_{b} \cap S_{c}$ are distinct $m$-1 element sets; so that their union has at least $m$ elements. As this union is contained in $S_{c}$ it coincides with $S_{c}$; and $S_{c} \subseteq S_{a} \cup S_{b}$. By the same token, with $c \neq d, S_{d}$ does not contain both $S_{a} \cap S_{c}$ and $S_{b} \cap S_{c}$; so that $S_{d}$ is contained in either $S_{a} \cup S_{c}$ or $S_{b} \cup S_{c}$. But this contradicts the assumption that $\left\{S_{a}\right\}$, and, hence, $\cup S_{a}$ are infinite.

Proposition 3.5. If $V$ is an isometric, linear mapping of an infinite-dimensional subspace $\mathscr{K}$ of $\mathscr{H}$ onto a set of product vectors in $\mathscr{H}_{m}^{(a)}$, then $\cap[V x]$ has dimension $m-1$. ([Vx] is the subspace of $H$ determined by $V x$.)

Proof. With $\left\{e_{j}\right\}$ an orthonormal basis for $\mathscr{K}, V e_{1}=x_{1} \wedge \cdots \wedge x_{m}$, $V e_{2}=y_{1} \wedge \cdots \wedge y_{m}$, and $V\left(e_{1}+e_{2}\right)=z_{1} \wedge \cdots \wedge z_{m}$, we have

$$
x_{1} \wedge \cdots \wedge x_{m}+y_{1} \wedge \cdots \wedge y_{m}=z_{1} \wedge \cdots \wedge z_{m} .
$$

Some $z_{j}$, say $z_{1}$, is not in $\left[x_{1}, \ldots, x_{m}\right]$. Thus $z_{1} \wedge x_{1} \wedge \cdots \wedge x_{m} \wedge y_{j}=0$; and $\left[y_{1}, \ldots, y_{m}\right] \subseteq\left[z_{1}, x_{1}, \ldots, x_{m}\right]$. Since $V e_{1}$ and $V e_{2}$ are not $0,\left[x_{1}, \ldots, x_{m}\right]$ and $\left[y_{1}, \ldots, y_{m}\right]$ are $m$-dimensional subspaces of $\left[z_{1}, x_{1}, \ldots, x_{m}\right]$, a space of dimension $m+1$. Thus $\left[x_{1}, \ldots, x_{m}\right] \cap\left[y_{1}, \ldots, y_{m}\right]$ has dimension at least $m-1$; and

$$
V e_{1}=x \wedge v_{1} \wedge \cdots \wedge v_{m-1}, V e_{2}=y \wedge v_{1} \wedge \cdots \wedge v_{m-1},
$$

with $\left\{x, v_{1}, \ldots, v_{m-1}\right\}$ and $\left\{y, v_{1}, \ldots, v_{m-1}\right\}$ orthonormal sets. In addition, $0=\left\langle V e_{1} \mid V e_{2}\right\rangle=\langle x \mid y\rangle \operatorname{det}\left(\left\langle v_{i} \mid v_{j}\right\rangle\right)=\langle x \mid y\rangle$. Hence, $E_{1}$ and $E_{2}$ commute, where $E_{j}$ is the orthogonal projection on $\mathscr{H}$ with range $\left[V e_{j}\right]$. Thus $\left\{E_{j}\right\}$ is an infinite, commuting family of projections on $\mathscr{H}$ such that $E_{j} E_{k}(\mathscr{H})$ has dimension $m-1$ when $j \neq k$. From Lemma $3.4, \bigcap_{j} E_{j}(\mathscr{H})$ has dimension $m-1$.

Lemma 3.6. If $V$ is a product isometry of $\mathscr{K}_{n}^{(a)}$ into $\mathscr{H}_{\mathscr{F}}^{(a)}$, with $\mathscr{K}$ a subspace of $\mathscr{H}$, then $V$ has range in some $\mathscr{H}_{m}^{(a)}$. If $n \leqq m$ and $\mathscr{K}$ is infinite dimensional then $\left[V\left(x_{1} \wedge \cdots \wedge x_{n}\right)\right] \cap\left[V\left(y_{1} \wedge \cdots \wedge y_{n}\right)\right]$ has dimension at least $m-n$.

Proof. If $\left\{e_{j}\right\}$ is an orthonormal basis for $\mathscr{K}$ and $\left\{i_{1}, \ldots, i_{t}\right\},\left\{j_{1}, \ldots, j_{t}\right\}$ are disjoint sets of indices,

$$
\begin{aligned}
1 & =\left\langle\left(e_{i_{1}}+e_{j_{1}}\right) \wedge e_{i_{2}} \wedge \cdots \wedge e_{i_{t}} \wedge e_{k_{t+1}} \wedge \cdots \wedge e_{k_{n}} \mid e_{i_{1}} \wedge \cdots \wedge e_{i_{t}} \wedge e_{k_{t+1}} \wedge \cdots \wedge e_{k_{n}}\right\rangle \\
& =\left\langle V\left(\left(e_{i_{1}}+e_{j_{1}}\right) \wedge e_{i_{2}} \wedge \cdots \wedge e_{i_{t}} \wedge e_{k_{t+1}} \wedge \cdots \wedge e_{k_{n}}\right) \mid V\left(e_{i_{1}} \wedge \cdots \wedge e_{i_{t}} \wedge e_{k_{t+1}} \wedge \cdots \wedge e_{k_{n}}\right)\right\rangle .
\end{aligned}
$$


Replacing $V\left(e_{i_{1}} \wedge \cdots \wedge e_{i_{t}} \wedge e_{k_{t+1}} \wedge \cdots \wedge e_{k_{n}}\right)$ by $V\left(e_{j_{1}} \wedge e_{i_{2}} \wedge \cdots \wedge e_{i_{t}} \wedge e_{k_{t+1}} \wedge \cdots \wedge e_{k_{n}}\right)$ in the preceding computation, it follows that both (product) vectors lie in the same space $\mathscr{H}_{m}^{(a)}$ as $V\left(\left(e_{i_{1}}+e_{j_{1}}\right) \wedge e_{i_{2}} \wedge \cdots \wedge e_{i_{t}} \wedge e_{k_{t+1}} \wedge \cdots \wedge e_{k_{n}}\right)$ does. Applying this result to successive replacements of $i_{2}, i_{3}, \ldots, i_{t}$ by $j_{2}, j_{3}, \ldots, j_{t}$, one concludes that $V\left(e_{j_{1}} \wedge \cdots \wedge e_{j_{t}} \wedge e_{k_{t+1}} \wedge \cdots \wedge e_{k_{n}}\right)$ lies in $\mathscr{H}_{m}^{(a)}$. Thus $V$ maps $\mathscr{K}_{n}^{(a)}$ into $\mathscr{H}_{m}^{(a)}$, since $\left\{e_{i_{1}} \wedge \cdots \wedge e_{i_{n}}\right\}$ is an orthonormal basis for $\mathscr{K}_{n}^{(a)}$.

Since $\left[x_{2}, \ldots, x_{n}\right]$ has dimension $n-1$, its orthogonal complement and $\left[y_{1}, \ldots, y_{n}\right]$ are not disjoint. Let $z_{1}$ be a unit vector in their intersection. Then $\left[V\left(z_{1} \wedge x_{2} \wedge \cdots \wedge x_{n}\right)\right] \cap\left[V\left(x_{1} \wedge \cdots \wedge x_{n}\right)\right]$ has dimension at least $m-1$, from Proposition 3.5; for $x \rightarrow V\left(x \wedge x_{2} \wedge \cdots \wedge x_{n}\right)$ is an isometric linear mapping of $\mathscr{K} \ominus\left[x_{2}, \ldots, x_{n}\right]$ onto a set of product vectors in $\mathscr{H}_{m}^{(a) 1}$. Let $z_{2}$ be a unit vector in $\left[y_{1}, \ldots, y_{n}\right]$ orthogonal to $\left[z_{1}, x_{3}, \ldots, x_{n}\right]$. Then

$$
\left[V\left(z_{1} \wedge x_{2} \wedge \cdots \wedge x_{n}\right)\right] \cap\left[V\left(z_{1} \wedge z_{2} \wedge x_{3} \wedge \cdots \wedge x_{n}\right)\right]
$$

has dimension at least $m-1$; so that its intersection with

$$
\left[V\left(z_{1} \wedge x_{2} \wedge \cdots \wedge x_{n}\right)\right] \cap\left[V\left(x_{1} \wedge \cdots \wedge x_{n}\right)\right]
$$

has dimension at least $m-2$ (both are subspaces of the $m$-dimensional space $\left.\left[V\left(z_{1} \wedge x_{2} \wedge \cdots \wedge x_{n}\right)\right]\right)$. Thus $\left[V\left(z_{1} \wedge z_{2} \wedge x_{3} \wedge \cdots \wedge x_{n}\right)\right] \cap\left[V\left(x_{1} \wedge \cdots \wedge x_{n}\right)\right]$ has dimension at least $m-2$. If we have found mutually orthogonal unit vectors $z_{1}, \ldots, z_{k-1}$ in $\left[y_{1}, \ldots, y_{n}\right]$ such that $\left[V\left(z_{1} \wedge \cdots \wedge z_{k-1} \wedge x_{k} \wedge \cdots \wedge x_{n}\right)\right]$ and $\left[V\left(x_{1} \wedge \cdots \wedge x_{n}\right)\right]$ have an intersection of dimension at least $m-k+1$, choose a unit vector $z_{k}$ in $\left[y_{1}, \ldots, y_{n}\right]$ orthogonal to $\left[z_{1}, \ldots, z_{k-1}, x_{k+1}, \ldots, x_{n}\right]$. Then $\left[V\left(z_{1} \wedge \cdots \wedge z_{k-1} \wedge x_{k} \wedge \cdots \wedge x_{n}\right)\right]$ and $\left[V\left(z_{1} \wedge \cdots \wedge z_{k} \wedge x_{k+1} \wedge \cdots \wedge x_{n}\right)\right]$ have intersection of dimension at least $m-1$. Thus $\left[V\left(z_{1} \wedge \cdots \wedge z_{k} \wedge x_{k+1} \wedge \cdots \wedge x_{n}\right)\right]$ and $\left[V\left(x_{1} \wedge \cdots \wedge x_{n}\right)\right]$ have intersection of dimension at least $m-k$. Finally, $z_{1} \wedge \cdots \wedge z_{n}=c y_{1} \wedge \cdots \wedge y_{n}$ and $\left[V\left(x_{1} \wedge \cdots \wedge x_{n}\right)\right] \cap\left[V\left(y_{1} \wedge \cdots \wedge y_{n}\right)\right]$ has dimension at least $m-n$.

Lemma 3.7. For each $n, \hat{U}$ maps $\mathscr{H}_{n}^{(a)}$ onto $\mathscr{H}_{n}^{(a)}$.

Proof. From Lemma 3.6, $\hat{U}$ maps $\mathscr{H}_{n}^{(a)}$ into some $\mathscr{H}_{m}^{(a)}$. Since $\hat{U}^{*}$ satisfies the same hypotheses as $\hat{U}, \hat{U}^{*}$ maps $\mathscr{H}_{m}^{(a)}$ into $\mathscr{H}_{n}^{(a)}$ (again, from Lemma 3.6). Thus $\hat{U}$ maps $\mathscr{H}_{n}^{(a)}$ onto $\mathscr{H}_{m}^{(a)}$. For some orthonormal sets $\left\{x_{1}, \ldots, x_{n}\right\}$ and $\left\{y_{1}, \ldots, y_{n}\right\}$ in $\mathscr{H}, \hat{U}\left(x_{1} \wedge \cdots \wedge x_{n}\right)=e_{1} \wedge \cdots \wedge e_{m}$ and $\hat{U}\left(y_{1} \wedge \cdots \wedge y_{n}\right)=e_{m+1} \wedge \cdots \wedge e_{2 m}$, where $\left\{e_{1}, \ldots, e_{2 m}\right\}$ is an orthonormal set in $\mathscr{H}$. If $n \leqq m$, from Lemma 3.6,

$$
\left[e_{1}, \ldots, e_{m}\right] \cap\left[e_{m+1}, \ldots, e_{2 m}\right](=(0))
$$

has dimension at least $m-n$. Thus $m \leqq n$. Applying this conclusion to $\hat{U}^{*}, n \leqq m$; so that $m=n$.

We denote the dimension of a (finite-dimensional) subspace $E$ of $\mathscr{H}$ by $d(E)$.

Proposition 3.8. If $V$ is a product isometry of $\mathscr{K}_{n}^{(a)}$ into $\mathscr{H}_{m}^{(a)}$, where $n \leqq m$ and $\mathscr{K}$ is an infinite-dimensional subspace of $\mathscr{H}$, and

$$
d\left(\left[V\left(e_{i_{1}} \wedge \cdots \wedge e_{i_{n}}\right)\right] \cap\left[V\left(e_{j_{1}} \wedge \cdots \wedge e_{j_{n}}\right)\right]\right)=m-n
$$

for some $e_{i_{1}}, \ldots, e_{i_{n}}, e_{j_{1}}, \ldots, e_{j_{n}}$, where $\left\{e_{j}\right\}$ is an orthonormal basis for $\mathscr{K}$, then $d\left(\bigcap_{x_{1}, \ldots, x_{n}}\left[V\left(x_{1} \wedge \cdots \wedge x_{n}\right)\right]\right)=m-n$.

\footnotetext{
1 Without loss of generality we assume that $\left\{x_{1}, \ldots, x_{n}\right\}$ and $\left\{y_{1}, \ldots, y_{n}\right\}$ are orthonormal sets.
} 
Proof. We write $E_{k}$ for $\left[V\left(e_{k_{1}} \wedge \cdots \wedge e_{k_{n}}\right)\right], E_{\bar{k} t}$ for

$$
\left[V\left(e_{k_{1}} \wedge \cdots \wedge e_{k_{r-1}} \wedge e_{t} \wedge e_{k_{r+1}} \wedge \cdots \wedge e_{k_{n}}\right)\right]
$$

and $F_{t}$ for $E_{i} \cap E_{j t}$. If $z$ lies in $\bigcap_{k} E_{k}$, then, with $x_{1}, \ldots, x_{n}$ in $\mathscr{K}, z \wedge V\left(x_{1} \wedge \cdots \wedge x_{n}\right)=0$, since $z \wedge V\left(e_{k_{1}} \wedge \cdots \wedge e_{k_{n}}\right)=0$ for all $k_{1}, \ldots, k_{n}$. Hence $z \in\left[V\left(x_{1} \wedge \cdots \wedge x_{n}\right)\right]$. It will suffice to show that $d\left(\bigcap_{k} E_{k}\right)=m-n$ (equivalently, that $E_{i} \cap E_{j} \subseteq E_{k}$ for each $k$ ). Proposition 3.5 establishes our result when $n=1$. Suppose we have proved it for values less than $n$.

Since $x_{1} \wedge \cdots \wedge x_{r-1} \wedge x_{r+1} \wedge \cdots \wedge x_{n} \rightarrow V\left(x_{1} \wedge \cdots \wedge x_{r-1} \wedge e_{i_{r}} \wedge x_{r+1} \wedge \cdots \wedge x_{n}\right)$ is an isometric, linear mapping of $\left(\mathscr{K} \ominus\left[e_{i_{r}}\right]\right)_{n-1}^{(a)}$ into $\mathscr{H}_{m}^{(a)} ; d\left(F_{i_{r}}\right) \geqq m-n+1$, from Lemma 3.6. If $d\left(F_{i_{r}}\right)>m-n+1$, then, since $d\left(E_{j} \cap E_{j i_{r}}\right)=m-1$ (from Proposition 3.5 - as argued in Lemma 3.6); $d\left(E_{i} \cap E_{j}\right)>m-n$, contrary to assumption. Thus $d\left(F_{i_{r}}\right)=m-n+1$. Our inductive hypothesis applies, and $d\left(\bigcap_{k_{1}, \ldots, k_{n}} E_{\bar{k} i_{r}}\right)$
$=m-n+1$. Since $\bigcap E_{\bar{k} i_{r} \subseteq E_{\bar{j} i_{r}}}$ $=m-n+1$. Since $\bigcap_{k_{1}, \ldots, k_{n}} E_{\bar{k} i_{r}} \cong E_{\bar{j} i_{r}}$;

$$
d\left(\left(\bigcap_{k_{1}, \ldots, k_{n}} E_{\bar{k} i_{r}}\right) \cap E_{j}\right)=d\left(\left(\bigcap_{k_{1}, \ldots, k_{n}} E_{\bar{k} i_{r}}\right) \cap E_{j} \cap E_{\bar{j} i_{r}}\right)=m-n=d\left(E_{i} \cap E_{j}\right) .
$$

But $\left(\bigcap_{k_{1}, \ldots, k_{n}}\right) E_{\bar{k} i_{r}} \cap E_{j} \subseteq E_{i} \cap E_{j}$. Thus

$$
E_{i} \cap E_{j} \subseteq \bigcap_{k_{1}, \ldots, k_{n}} E_{\bar{k} i_{r}} \subseteq F_{i_{r}}
$$

In particular, we have established (under the induction hypothesis) that if $d\left(E_{i} \cap E_{j}\right)=m-n$ then $E_{i} \cap E_{j} \subseteq E_{k}$ provided one of the $k_{1}, \ldots, k_{n}$ is in $\left\{i_{1}, \ldots, i_{n}, j_{1}, \ldots, j_{n}\right\}$. (In our argument $k_{r}=i_{r}$ ).

Having proved that $d\left(F_{i_{r}}\right)=m-n+1$ and $E_{i} \cap E_{j} \subseteq F_{i_{r}}$, it follows that $F_{i_{r}}$ is generated by $E_{i} \cap E_{j}$ and a unit vector $f_{r}$ orthogonal to it. Moreover $\left\{f_{1}, \ldots, f_{n}\right\}$ are linearly independent. To see this, note that $\bigcap_{t} E_{j t}$ is $m-1$ dimensional, from Proposition 3.5 , so that $E_{\bar{j} t}$ is generated by $\bigcap_{t} E_{\bar{j} t}$ and a unit vector $g_{t}$ orthogonal to $\bigcap_{t} E_{\bar{j} t}$. In addition,

$$
\begin{aligned}
0 & =\left\langle V\left(e_{j_{1}} \wedge \cdots \wedge e_{j_{r-1}} \wedge e_{t} \wedge e_{j_{r+1}} \wedge \cdots \wedge e_{j_{n}}\right) \mid V\left(e_{j_{1}} \wedge \cdots \wedge e_{j_{r-1}} \wedge e_{t^{\prime}} \wedge e_{j_{r+1}} \wedge \cdots \wedge e_{j_{n}}\right)\right\rangle \\
& =\left\langle g_{t} \mid g_{t^{\prime}}\right\rangle .
\end{aligned}
$$

Thus no $E_{\bar{j} t}$ is contained in the union of the others (for $g_{t}$ is orthogonal to that union). Now $f_{r}$ is not in $\bigcap_{t} E_{\bar{j} t}$; for, otherwise $f_{r}$ is in $E_{j}$, hence, in $E_{i} \cap E_{j}$, contrary to the choice of $f_{r}$. Thus $f_{r}$ and $\bigcap_{t} E_{\bar{j} t}$ generate $E_{\bar{j} i_{r}}$; so that a linear relation among $\left\{f_{1}, \ldots, f_{n}\right\}$ would entail that some $E_{j t}$ is contained in the union of the others. By the same token, if $F_{t}$ has dimension $m-n+1$ or greater and $t$ is not in $\left\{i_{1}, \ldots, i_{n}\right\}, F_{t}$ contains a unit vector $f_{0}$ orthogonal to $E_{i} \cap E_{j}$ and linearly independent of $\left\{f_{1}, \ldots, f_{n}\right\}$. Recalling that $E_{i} \cap E_{j} \subseteq F_{t}$ (as established before), and $F_{t} \cong E_{i}$ (by 
definition of $\left.F_{t}\right)$; we see that $d\left(E_{i}\right)$ would exceed $m$. Thus $d\left(F_{t}\right)=m-n$ when $t \notin\left\{i_{1}, \ldots, i_{n}\right\}$; so that $E_{i} \cap E_{j} \subseteq F_{k_{r}} \subseteq E_{k}$. (We now have that

$$
d\left(\left[V\left(e_{i_{1}} \wedge \cdots \wedge e_{i_{n}}\right)\right] \cap\left[V\left(e_{j_{1}} \wedge \cdots \wedge e_{j_{r-1}} \wedge e_{k_{r}} \wedge e_{j_{r+1}} \wedge \cdots \wedge e_{j_{n}}\right)\right]\right)=m-n
$$

when $k_{r} \notin\left\{i_{1}, \ldots, i_{n}\right\}$; and, from the first part of this proof,

since

$$
F_{k_{r}} \subseteq E_{k}=\left[V\left(e_{k_{1}} \wedge \cdots \wedge e_{k_{n}}\right)\right]
$$

$$
k_{r} \in\left\{i_{1}, \ldots, i_{n}, j_{1}, \ldots, j_{r-1}, k_{r}, j_{r+1}, \ldots, j_{n}\right\} \text {.) }
$$

Lemma 3.9. The equality,

$$
\begin{aligned}
& d\left(\left[x_{1}, \ldots, x_{n}\right] \cap \cdots \cap\left[z_{1}, \ldots, z_{n}\right]\right) \\
& \quad=d\left(\left[\hat{U}\left(x_{1} \wedge \cdots \wedge x_{n}\right)\right] \cap \cdots \cap\left[\hat{U}\left(z_{1} \wedge \cdots \wedge z_{n}\right)\right]\right)
\end{aligned}
$$

is valid for finite and infinite intersections.

Proof. We establish the assertion of the lemma, first, for the intersection $\left[x_{1}, \ldots, x_{n}\right] \cap\left[z_{1}, \ldots, z_{n}\right]$ of two $n$-dimensional subspaces of $\mathscr{H}$. If $\left\{v_{1}, \ldots, v_{k}\right\}$ is an orthonormal basis for this intersection, changing notation, we can write $x_{1} \wedge \cdots \wedge x_{n-k} \wedge v_{1} \wedge \cdots \wedge v_{k}$ and $z_{1} \wedge \cdots \wedge z_{n-k} \wedge v_{1} \wedge \cdots \wedge v_{k}$ in place of $x_{1} \wedge \cdots \wedge x_{n}$ and $z_{1} \wedge \cdots \wedge z_{n}$. In Lemma 3.7 we noted that $\hat{U}$ maps $\mathscr{H}_{n}^{(a)}$ into $\mathscr{H}_{n}^{(a)}$; so that $y_{1} \wedge \cdots \wedge y_{n-k} \rightarrow \hat{U}\left(y_{1} \wedge \cdots \wedge y_{n-k} \wedge v_{1} \wedge \cdots \wedge v_{k}\right)$ is a product isometry of $\left(\mathscr{H} \ominus\left[v_{1}, \ldots, v_{k}\right]\right)_{n-k}^{(a)}$ into $\mathscr{H}_{n}^{(a)}$. From Lemma 3.6,

$$
\left[\hat{U}\left(x_{1} \wedge \cdots \wedge x_{n}\right)\right] \cap\left[\hat{U}\left(z_{1} \wedge \cdots \wedge z_{n}\right)\right]
$$

has dimension at least $k$. Applying this to $\hat{U}^{-1}$, we see that

$$
d\left(\left[\hat{U}\left(x_{1} \wedge \cdots \wedge x_{n}\right)\right] \cap\left[\hat{U}\left(z_{1} \wedge \cdots \wedge z_{n}\right)\right]\right)=k=d\left(\left[x_{1}, \ldots, x_{n}\right] \cap\left[z_{1}, \ldots, z_{n}\right]\right) .
$$

Let $w_{1}, \ldots, w_{r}$ be an orthonormal basis for $\left[x_{1}, \ldots, x_{n}\right] \cap \cdots \cap\left[z_{1}, \ldots, z_{n}\right]$; and let $u_{1}, \ldots, u_{n-r}, u_{1}^{\prime}, \ldots, u_{n-r}^{\prime}$ be an orthonormal set of vectors in $\mathscr{H} \ominus\left[w_{1}, \ldots, w_{r}\right]$. Then, from the preceding,

$$
d\left(\left[\hat{U}\left(u_{1} \wedge \cdots \wedge u_{n-r} \wedge w_{1} \wedge \cdots \wedge w_{r}\right)\right] \cap\left[\hat{U}\left(u_{1}^{\prime} \wedge \cdots \wedge u_{n-r}^{\prime} \wedge w_{1} \wedge \cdots \wedge w_{r}\right)\right]\right)=r .
$$

Moreover $y_{1} \wedge \cdots \wedge y_{n-r} \rightarrow \hat{U}\left(y_{1} \wedge \cdots \wedge y_{n-r} \wedge w_{1} \wedge \cdots \wedge w_{r}\right)$ is a product isometry of $\left(\mathscr{H} \ominus\left[w_{1}, \ldots, w_{r}\right]\right)_{n-r}^{(a)}$ into $\mathscr{H}_{n}^{(a)}$. From Proposition 3.8,

$$
d\left(\bigcap_{y_{1}, \ldots, y_{n-r}}\left[\hat{U}\left(y_{1} \wedge \cdots \wedge y_{n-r} \wedge w_{1} \wedge \cdots \wedge w_{r}\right)\right]\right)=r .
$$

Thus $d\left(\left[\hat{U}\left(x_{1} \wedge \cdots \wedge x_{n}\right)\right] \cap \cdots \cap\left[\hat{U}\left(z_{1} \wedge \cdots \wedge z_{n}\right)\right]\right) \geqq r$. Applying this to $\hat{U}^{-1}$, we have

$$
\begin{aligned}
& r=d\left(\left[x_{1} \wedge \cdots \wedge x_{n}\right] \cap \cdots \cap\left[z_{1} \wedge \cdots \wedge z_{n}\right]\right) \\
& \quad \geqq d\left(\left[\hat{U}\left(x_{1} \wedge \cdots \wedge x_{n}\right)\right] \cap \cdots \cap\left[\hat{U}\left(z_{1} \wedge \cdots \wedge z_{n}\right)\right]\right),
\end{aligned}
$$

from which our lemma follows.

Corollary 3.10. With $e_{0}$ a unit vector in $\mathscr{H}$,

$$
d\left(\bigcap_{x_{1}, \ldots, x_{n-1}}\left[\hat{U}\left(x_{1} \wedge \cdots \wedge x_{n-1} \wedge e_{0}\right)\right]\right)=1
$$


Lemma 3.11. For each unit vector $e_{0}$ in $\mathscr{H}, \hat{U} e_{0}$ lies in

$$
\bigcap_{x_{1}, \ldots, x_{n-1}}\left[\hat{U}\left(x_{1} \wedge \cdots \wedge x_{n-1} \wedge e_{0}\right)\right] .
$$

Proof. From Corollary 3.10, there is a unit vector $f_{1}$ which generates $\bigcap\left[\hat{U}\left(x_{1} \wedge \cdots \wedge x_{n-1} \wedge e_{0}\right)\right]$. Thus $\hat{U}\left(x_{1} \wedge \cdots \wedge x_{n-1} \wedge e_{0}\right)=x_{1}^{\prime} \wedge \cdots \wedge x_{n-1}^{\prime} \wedge f_{1}$. $\stackrel{x_{1}, \ldots, x_{n-1}}{\text { Now } d}\left(_{y_{1}, \ldots, y_{n-1}}\right.$

$$
\begin{aligned}
& \left.\left[\hat{U}^{*}\left(y_{1} \wedge \cdots \wedge y_{n-1} \wedge f_{1}\right)\right]\right)=1, \text { from Corollary } \\
& 1=d\left(\bigcap_{x_{1}, \ldots, x_{n-1}}\left[\hat{U}^{*} \hat{U}\left(x_{1} \wedge \cdots \wedge x_{n-1} \wedge e_{0}\right)\right]\right) \\
& \quad=d\left(\bigcap_{x_{1}, \ldots, x_{n-1}}\left[\hat{U}^{*}\left(x_{1}^{\prime} \wedge \cdots \wedge x_{n-1}^{\prime} \wedge f_{1}\right)\right]\right) \\
& \geqq d\left(\bigcap_{y_{1}, \ldots, y_{n-1}}\left[\hat{U}^{*}\left(y_{1} \wedge \cdots \wedge y_{n-1} \wedge f_{1}\right)\right]\right)=1 ;
\end{aligned}
$$

so that each $\left[\hat{U}^{*}\left(y_{1} \wedge \cdots \wedge y_{n-1} \wedge f_{1}\right)\right]$ contains $e_{0}$. Thus

$$
\hat{U}^{*}\left(y_{1} \wedge \cdots \wedge y_{n-1} \wedge f_{1}\right)=y_{1}^{\prime} \wedge \cdots \wedge y_{n-1}^{\prime} \wedge e_{0} .
$$

Suppose $f_{1}$ is not a scalar multiple of $\hat{U} e_{0}\left(=e_{1}\right)$. Then $\left\langle f_{1} \mid e_{2}\right\rangle \neq 0$, where $e_{2}$ is a unit vector in $\left[e_{1}, f_{1}\right]$ orthogonal to $e_{1}$. Let $\left\{e_{j}\right\}_{j=1,2, \ldots}$ be an orthonormal basis for $\mathscr{H}$; and let $A$ be $\hat{U} a\left(e_{0}\right)^{*} a\left(e_{0}\right) \hat{U}^{*}$. Then

$$
\begin{aligned}
A\left(f_{1} \wedge e_{j_{2}} \wedge \cdots \wedge e_{j_{n}}\right) & =\hat{U} a\left(e_{0}\right)^{*} a\left(e_{0}\right)\left(e_{0} \wedge e_{j_{2}}^{\prime} \wedge \cdots \wedge e_{j_{n}}^{\prime}\right) \\
& =f_{1} \wedge e_{j_{2}} \wedge \cdots \wedge e_{j_{n}} .
\end{aligned}
$$

Finite sums, $\Sigma c_{i_{1} \ldots i_{p} ; j_{1} \ldots j_{q}} a\left(e_{i_{1}}\right)^{*} \ldots a\left(e_{i_{p}}\right) * a\left(e_{j_{1}}\right) \ldots a\left(e_{j_{q}}\right)(=B)$, form a normdense subset of $\mathfrak{A}$. Let $\varepsilon$ be $\left|\left\langle f_{1} \mid e_{2}\right\rangle\right| / 5$, and choose $B$ such that $\|A-B\|<\varepsilon$. Then

$$
\varepsilon^{2}>\left\|(A-B) e_{1}\right\|^{2} \geqq\left|c_{2 ; 1}\right|^{2},
$$

(examining the coefficient of $e_{2}$ ),

$$
\varepsilon^{2}>\left\|(A-B) e_{m}\right\|^{2} \geqq\left|c_{0}\right|^{2},
$$

where $c_{0}$ is the coefficient of $I$ in the sum representation of $B$ and all $e_{i}, e_{j}$ appearing in this sum are among $e_{1}, \ldots, e_{m-1}$ (so that $A$ and all terms of $B$ other than $c_{0} I$ map $e_{m}$ to 0 or a multiple of a basis element other than $e_{m}$ ), and

$$
\varepsilon^{2}>\left\|(A-B) e_{2}\right\|^{2} \geqq\left|c_{0}+c_{2 ; 2}\right|^{2}
$$

(examining the coefficient of $e_{2}$ ).

$$
\text { Thus } \quad\left|c_{0}\right|<\varepsilon, \quad\left|c_{2 ; 1}\right|<\varepsilon \quad \text { and } \quad\left|c_{2 ; 2}\right|<2 \varepsilon \text {. }
$$

The only terms in the sum for $B$ that yield multiples of $e_{2} \wedge e_{m+2} \wedge \cdots \wedge e_{m+n}$ when $B$ acts on

$$
\begin{aligned}
& f_{1} \wedge e_{m+2} \wedge \cdots \wedge e_{m+n} \\
& \quad\left(=\left\langle e_{1} \mid f_{1}\right\rangle e_{1} \wedge e_{m+2} \wedge \cdots \wedge e_{m+n}+\left\langle e_{2} \mid f_{1}\right\rangle e_{2} \wedge e_{m+2} \wedge \cdots \wedge e_{m+n}\right)
\end{aligned}
$$


are $c_{0} I, c_{2 ; 1} a^{*}\left(e_{2}\right) a\left(e_{1}\right)$, and $c_{2 ; 2} a^{*}\left(e_{2}\right) a\left(e_{2}\right)$. Thus

$$
\begin{aligned}
\left|\left\langle f_{1} \mid e_{2}\right\rangle\right|^{2} / 25 & =\varepsilon^{2} \\
& \geqq\left\|(A-B)\left(f_{1} \wedge e_{m+2} \wedge \cdots \wedge e_{m+n}\right)\right\|^{2} \\
& \geqq\left|\left\langle e_{2} \mid f_{1}\right\rangle-c_{0}\left\langle e_{2} \mid f_{1}\right\rangle-c_{2 ; 1}\left\langle e_{1} \mid f_{1}\right\rangle-c_{2 ; 2}\left\langle e_{2} \mid f_{1}\right\rangle\right|^{2}
\end{aligned}
$$

so that

$$
\begin{aligned}
\left|\left\langle f_{1} \mid e_{2}\right\rangle\right| / 5 & \\
& \geqq\left|\left\langle f_{1} \mid e_{2}\right\rangle\right|-\left|c_{0}\right|\left|\left\langle f_{1} \mid e_{2}\right\rangle\right|-\left|c_{2 ; 1}\right|\left|\left\langle f_{1} \mid e_{1}\right\rangle\right|-\left|c_{2 ; 2}\right|\left|\left\langle f_{1} \mid e_{2}\right\rangle\right| \\
& >\left|\left\langle f_{1} \mid e_{2}\right\rangle\right|-4 \varepsilon=\left|\left\langle f_{1} \mid e_{2}\right\rangle\right| / 5
\end{aligned}
$$

a contradiction. Thus $f_{1}=c^{\prime} e_{1}$ for some scalar $c^{\prime}$.

Lemma 3.12. There is a sequence $\left\{c_{n}\right\}$ of complex scalars of modulus 1 such that $\hat{U}\left(x_{1} \wedge \cdots \wedge x_{n}\right)=c_{n}\left(U x_{1} \wedge \cdots \wedge U x_{n}\right)$, where $U$ is the restriction of $\hat{U}$ to oneparticle space.

Proof. Let $\left\{e_{j}\right\}$ be an orthonormal basis for $\mathscr{H}$. Then

$$
U e_{j} \in \bigcap_{x_{1}, \ldots, x_{n-1}}\left[\hat{U}\left(x_{1} \wedge \cdots \wedge x_{n-1} \wedge e_{j}\right)\right]
$$

for all $j$ and $n$. Thus $\hat{U}\left(e_{j_{1}} \wedge \cdots \wedge e_{j_{n}}\right)$ and $U e_{j_{1}} \wedge \cdots \wedge U e_{j_{n}}$ differ by a phase factor. Say

and

$$
\hat{U}\left(e_{j_{1}} \wedge \cdots \wedge e_{j_{n}}\right)=c U e_{j_{1}} \wedge \cdots \wedge U e_{j_{n}}
$$

$$
\hat{U}\left(e_{i_{1}} \wedge e_{j_{2}} \wedge \cdots \wedge e_{j_{n}}\right)=c^{\prime} U e_{i_{1}} \wedge U e_{j_{2}} \wedge \cdots \wedge U e_{j_{n}} .
$$

Writing $f$ for $\left(e_{j_{1}}+e_{i_{1}}\right) / \sqrt{2}$ and $f^{\prime}$ for $\left(c e_{j_{1}}+c^{\prime} e_{i_{1}}\right) / \sqrt{2}, \hat{U}\left(f \wedge e_{j_{2}} \wedge \cdots \wedge e_{j_{n}}\right)$ $=U f^{\prime} \wedge U e_{j_{2}} \wedge \cdots \wedge U e_{j_{n}}$. Since $\hat{U}\left(f \wedge e_{j_{2}} \wedge \cdots \wedge e_{j_{n}}\right)=c^{\prime \prime} U f \wedge U e_{j_{2}} \wedge \cdots \wedge U e_{j_{n}}$ and $U f^{\prime}-c^{\prime \prime} U f$ is orthogonal to $U e_{j_{2}}, \ldots, U e_{j_{n}}$; we have $U f^{\prime}=U c^{\prime \prime} f$. Thus $c e_{j_{1}}+c^{\prime} e_{i_{1}}=c^{\prime \prime} e_{j_{1}}+c^{\prime \prime} e_{i_{1}}$; and $c=c^{\prime \prime}=c^{\prime}$. Changing basis elements successively, we conclude that $\hat{U}\left(e_{j_{1}} \wedge \cdots \wedge e_{j_{n}}\right)=c U e_{j_{1}} \wedge \cdots \wedge U e_{j_{n}}$, for all $j_{1}, \ldots, j_{n}$. Writing $c_{n}$ for $c$, we have $\hat{U}\left(x_{1} \wedge \cdots \wedge x_{n}\right)=c_{n}\left(U x_{1} \wedge \cdots \wedge U x_{n}\right)$. Of course $c_{1}=1$; and $\hat{U} \Phi_{0}=\Phi_{0}$.

Lemma 3.13. For all $x_{1}, \ldots, x_{n}$ in $\mathscr{H}$ and $n=0,1,2, \ldots, \hat{U}\left(x_{1} \wedge \ldots \wedge x_{n}\right)$ $=U x_{1} \wedge \cdots \wedge U x_{n}$.

Proof. From Lemma 3.12, there is a sequence $\left\{c_{n}\right\}$ of scalars of modulus 1 such that $\hat{U}\left(x_{1} \wedge \cdots \wedge x_{n}\right)=c_{n}\left(U x_{1} \wedge \cdots \wedge U x_{n}\right)$. If $\left\{e_{j}\right\}$ is an orthonormal basis for $\mathscr{H}$, so is $\left\{U e_{j}\right\}$. Write $f_{j}$ for $U e_{j}$. Since $\hat{U} a\left(e_{j}\right) U^{*}\left(f_{j} \wedge f_{j_{2}} \wedge \cdots \wedge f_{j_{n}}\right)=\bar{c}_{n} \hat{U}\left(e_{j_{2}} \wedge \cdots \wedge e_{j_{n}}\right)$ $=c_{n-1} \bar{c}_{n}\left(f_{j_{2}} \wedge \cdots \wedge f_{j_{n}}\right)$ and $\hat{U} a\left(e_{j}\right) \hat{U}^{*}\left(f_{j_{1}} \wedge \ldots \wedge f_{j_{n}}\right)=0$ if $j \notin\left\{j_{1}, \ldots, j_{n}\right\}$; we have that $\hat{U} a\left(e_{j}\right) \hat{U}^{*}\left|\mathscr{H}_{n}^{(a)}=c_{n-1} \bar{c}_{n} a\left(f_{j}\right)\right| \mathscr{H}_{n}^{(a)}$.

Writing $c_{n}^{\prime}$ for $c_{n-1} \bar{c}_{n}$ (so that $c_{1}^{\prime}=1$ ), we show that $c_{n}^{\prime}=c_{n-1}^{\prime}=\cdots=1$. Let $\Sigma c_{i_{1} \ldots i_{p} ; j_{1} \ldots j_{2}} a\left(f_{i_{1}}\right)^{*} \ldots a\left(f_{i_{p}}\right)^{*} a\left(f_{j_{1}}\right) \ldots a\left(f_{j_{q}}\right) \quad(=B)$ be chosen such that $\|A-B\|<\varepsilon$, where $A=\hat{U} a\left(e_{1}\right) \hat{U}^{*}$. Suppose $i_{1}, \ldots, i_{p} ; j_{1}, \ldots, j_{q}$ are less than $m$. Then, examining the coefficient of $f_{m+2} \wedge \cdots \wedge f_{m+n}$,

$$
\varepsilon^{2}>\left\|(A-B)\left(f_{1} \wedge f_{m+2} \wedge \cdots \wedge f_{m+n}\right)\right\|^{2} \geqq\left|c_{n}^{\prime}-c_{0 ; 1}\right|^{2} .
$$

This inequality holds for $n=1,2, \ldots$, so that $\left|c_{n}^{\prime}-c_{n-1}^{\prime}\right|<2 \varepsilon$ for all positive $\varepsilon$. Thus $1=c_{1}^{\prime}=c_{2}^{\prime}=\cdots$, and $c_{n}=c_{n-1}=\cdots=c_{0}=1$. 
Proof of Theorem 3.1. We know that $\hat{U}$ implements $\alpha$ (Lemma 3.3) and is the product unitary operator on $\mathscr{H}_{\mathscr{F}}^{(a)}$ corresponding to $U$ on $\mathscr{H}$, when $\hat{\alpha}\left(\phi_{0}\right)=\phi_{0}$ (Lemma 3.13). The case in which $\hat{\alpha}\left(\phi_{0}\right)=\phi_{I}$ is reduced to this case following the proof of Lemma 3.2. To conclude the proof, we note that $\alpha(a(f))=a(U f)$, or, equivalently, that $\alpha\left(a(f)^{*}\right)=a(U f)^{*}$. For this, observe that

$$
\begin{aligned}
\alpha\left(a(f)^{*}\right) & \left(f_{1} \wedge \cdots \wedge f_{n}\right) \\
= & \hat{U} a(f)^{*} \hat{U}^{*}\left(f_{1} \wedge \cdots \wedge f_{n}\right) \\
= & \hat{U} a(f)^{*}\left(U^{*} f_{1} \wedge \cdots \wedge U^{*} f_{n}\right) \\
& =\hat{U}\left(f \wedge U^{*} f_{1} \wedge \cdots \wedge U^{*} f_{n}\right) \\
& =(U f) \wedge f_{1} \wedge \cdots \wedge f_{n} \\
& =a(U f)^{*}\left(f_{1} \wedge \cdots \wedge f_{n}\right),
\end{aligned}
$$

for all $f_{1}, \ldots, f_{n}$ in $\mathscr{H}$. Thus $\alpha\left(a(f)^{*}\right)=a(U f)^{*}$.

\section{Product Unitary Operators on $n$-Particle Space}

In the preceding section, after showing (Lemma 3.3) that $\hat{U}$ is a product unitary operator on Fock space, we make no further use of the hypothesis that $\hat{U}$ induces an automorphism of $\mathfrak{U}$ until Lemma 3.11. With this hypothesis, it is proved (Lemma 3.13) that $\hat{U}$ is induced by a unitary operator on one-particle space. The fact that this same result is valid for a product unitary operator defined only on $n$-particle space is proved in the theorem that follows.

Theorem 4.1. If $\hat{U}$ is a product unitary operator on n-particle space $\mathscr{H}_{n}^{(a)}$, there is a unitary operator $U$ on one-particle space $\mathscr{H}$ such that $\hat{U}\left(x_{1} \wedge \cdots \wedge x_{n}\right)$ $=U x_{1} \wedge \cdots \wedge U x_{n}$.

We prove this theorem with the aid of the following lemmas (notation as in Theorem 4.1). Again, the argument will justify the use of the notation $\hat{U}$.

Lemma 4.2. If $\left\{e_{j}\right\}$ is an orthonormal basis for $\mathscr{H}$, there is a unit vector $f_{j}$ in $\bigcap_{x_{1}, \ldots, x_{n-1}}\left[\hat{U}\left(x_{1} \wedge \cdots \wedge x_{n-1} \wedge e_{j}\right)\right]$ such that $\left\{f_{j}\right\}$ is an orthonormal basis for $\mathscr{H}$.

Proof. To show that $\left\langle f_{j} \mid f_{k}\right\rangle=0$, when $j \neq k$, we may assume that $j$ and $k$ are in $\{1, \ldots, n+1\}$. Let $E_{j}$ be the $n$-dimensional space,

$$
\left[\hat{U}\left(e_{1} \wedge \cdots \wedge e_{j-1} \wedge e_{j+1} \wedge \cdots \wedge e_{n+1}\right)\right], j=1, \ldots, n+1,
$$

and $E$ be the space $E_{1} \vee E_{2}$. From Lemma 3.9, $E_{1} \cap E_{2}, E_{1} \cap E_{j}$, and $E_{2} \cap E_{j}$ are $n-1$ dimensional; and $E_{1} \cap E_{2} \cap E_{j}$ is $n-2$ dimensional, when $j \neq 1,2$. Thus $E$ is $n+1$ dimensional; and $E_{1}, E_{2}$ contain unit vectors $v_{1}, v_{2}$, respectively, that lie in $E_{j}$ but not in $E_{1} \cap E_{2} \cap E_{j}$. It follows that $E_{1} \cap E_{2} \cap E_{j}$ and $v_{1}$ generate an $n-1$ dimensional subspace of $E_{1} \cap E_{j}$ (which is, therefore, $E_{1} \cap E_{j}$ ). As $v_{2}$ is in $E_{2}$ but not in $E_{1} \cap E_{2} \cap E_{j}, v_{2}$ is not in $E_{1} \cap E_{j}$; so that $E_{1} \cap E_{2} \cap E_{j}, v_{1}$ and $v_{2}$ generate an $n$-dimensional subspace of $E_{j}$, which is therefore, $E_{j}$. Thus $E_{j}$ is a subspace of $E$. Let $f_{j}$ be a unit vector in $E$ (unique up to a phase factor) orthogonal to $E_{j}$. From Section 2 and Lemma 3.9, $E_{j}$ and $E_{k}$ are perpendicular, when $j \neq k$; and both are subspaces of $E$. Hence $f_{j}$ is in $E_{k}$ and $f_{k}$ is in $E_{j}$, when $j \neq k$. Since $f_{j}$ is orthogonal 
to $E_{j} ;\left\langle f_{j} \mid f_{k}\right\rangle=0$, when $j \neq k$. Thus $\left\{f_{1}, \ldots, f_{n+1}\right\}$ is an orthonormal basis for $E$, and $E_{j}=\left[f_{1}, \ldots, f_{j-1}, f_{j+1}, \ldots, f_{n+1}\right]$. Hence $f_{j}$ generates $\bigcap_{k \neq j} E_{k}$. From Corollary 3.10, $\bigcap\left[\hat{U}\left(x_{1} \wedge \cdots \wedge x_{n-1} \wedge e_{j}\right)\right] \quad$ is a one-dimensional space (contained in $\bigcap_{k \neq j}^{x_{1}, \ldots, x_{n}-1} E_{k}$ ); so that $f_{j}$ generates it.

Lemma 4.3. If $\hat{U}$ is a product unitary operator on $\mathscr{H}_{n}^{(a)}$ and $\left\{e_{j}\right\}$ is an orthonormal basis for $\mathscr{H}$, there is an orthonormal basis $\left\{f_{j}\right\}$ for $\mathscr{H}$ such that $\hat{U}\left(e_{i_{1}} \wedge \cdots \wedge e_{i_{n}}\right)$ $=f_{i_{1}} \wedge \cdots \wedge f_{i_{n}}$ for all $i_{1}, \ldots, i_{n}$.

Proof. From Lemma 4.2, we can find an orthonormal basis $\left\{f_{j}^{\prime}\right\}$ such that $f_{j}^{\prime} \in \bigcap\left[\hat{U}\left(x_{1} \wedge \cdots \wedge x_{n-1} \wedge e_{j}\right)\right]$ for all $j$. Thus $\hat{U}\left(e_{i_{1}} \wedge \cdots \wedge e_{i_{n}}\right)$ $=c_{i_{1} \ldots i_{n}} f_{i_{1}}^{x_{1}, \ldots, x_{n-1}} \wedge \wedge \wedge f_{i_{n}}^{\prime}$, where $\left|c_{i_{1} \ldots i_{n}}\right|=1$. Let $c_{j}^{\prime}$ be $c_{1 \ldots j-1 j+1 \ldots n+1}$, and let $c_{j}$ be $\bar{c}_{j}^{\prime}\left(\prod_{k=1}^{n+1} c_{k}^{\prime}\right)^{1 / n}$. With $f_{j}$ as $c_{j} f_{j}^{\prime}$

$$
\begin{aligned}
\hat{U}\left(e_{1} \wedge\right. & \left.\cdots \wedge e_{j-1} \wedge e_{j+1} \wedge \cdots \wedge e_{n+1}\right) \\
& =c_{j}^{\prime} f_{1}^{\prime} \wedge \cdots \wedge f_{j-1}^{\prime} \wedge f_{j+1}^{\prime} \wedge \cdots \wedge f_{n+1}^{\prime} \\
& =c_{j}^{\prime} \overline{\left(c_{1} \ldots c_{j-1} c_{j+1} \ldots c_{n+1}\right)} f_{1} \wedge \cdots \wedge f_{j-1} \wedge f_{j+1} \wedge \cdots \wedge f_{n+1} \\
& =f_{1} \wedge \cdots \wedge f_{j-1} \wedge f_{j+1} \wedge \cdots \wedge f_{n+1} .
\end{aligned}
$$

Suppose, now, that we have chosen $f_{1}, \ldots, f_{m}, m>n$, so that $f_{j}$ is a multiple of $f_{j}^{\prime}$ and $\hat{U}\left(e_{i_{1}} \wedge \cdots \wedge e_{i_{n}}\right)=f_{i_{1}} \wedge \cdots \wedge f_{i_{n}}$ when $1 \leqq i_{1}<i_{2}<\cdots<i_{n} \leqq m$. Suppose $\hat{U}\left(e_{1} \wedge \cdots \wedge e_{n-1} \wedge e_{m+1}\right)=c^{\prime} f_{1} \wedge \cdots \wedge f_{n-1} \wedge f_{m+1}^{\prime}$ and $\hat{U}\left(e_{2} \wedge \cdots \wedge e_{n} \wedge e_{m+1}\right)$ $=c^{\prime \prime} f_{2} \wedge \cdots \wedge f_{n} \wedge f_{m+1}^{\prime}$. From Lemma 4.2, there is a vector $f$ such that

and

$$
\hat{U}\left(e_{1} \wedge \cdots \wedge e_{n-1} \wedge\left(e_{m+1}+e_{n+1}\right)\right)=f_{1} \wedge \cdots \wedge f_{n-1} \wedge f
$$

$$
\hat{U}\left(e_{2} \wedge \cdots \wedge e_{n} \wedge\left(e_{m+1}+e_{n+1}\right)\right)=c f_{2} \wedge \cdots \wedge f_{n} \wedge f .
$$

Since

and

$$
\hat{U}\left(e_{1} \wedge \cdots \wedge e_{n-1} \wedge\left(e_{m+1}+e_{n+1}\right)\right)=f_{1} \wedge \cdots \wedge f_{n-1} \wedge\left(c^{\prime} f_{m+1}^{\prime}+f_{n+1}\right)
$$

$$
\hat{U}\left(e_{2} \wedge \cdots \wedge e_{n} \wedge\left(e_{m+1}+e_{n+1}\right)\right)=f_{2} \wedge \cdots \wedge f_{n} \wedge\left(c^{\prime \prime} f_{m+1}^{\prime}+f_{n+1}\right) ;
$$

$f=c^{\prime} f_{m+1}^{\prime}+f_{n+1}$ and $c f=c^{\prime \prime} f_{m+1}^{\prime}+f_{n+1}$. Thus $\left(c c^{\prime}-c^{\prime \prime}\right) f_{m+1}^{\prime}+(c-1) f_{n+1}=0$; and $c=1, c^{\prime}=c^{\prime \prime}$.

Applying this conclusion to step-by-step replacements, we have that $\hat{U}\left(e_{i_{1}} \wedge \cdots \wedge e_{i_{n}} \wedge e_{m+1}\right)=c f_{i_{1}} \wedge \cdots \wedge f_{i_{n}} \wedge f_{m+1}^{\prime}$ for one phase factor $c$ and all $i_{1}, \ldots, i_{n}$ less than $m+1$. Defining $f_{m+1}$ to be $c f_{m+1}^{\prime}$, our induction yields the basis $\left\{f_{j}\right\}$.

Proof of Theorem 4.1. With $\left\{e_{j}\right\}$ and $\left\{f_{j}\right\}$ as in Lemma 4.3, let $U$ be the unitary operator on $\mathscr{H}$ for which $U e_{j}=f_{j}, j=1,2, \ldots$ Then $\hat{U}\left(e_{i_{1}} \wedge \ldots \wedge e_{i_{n}}\right)$ $=U e_{i_{1}} \wedge \cdots \wedge U e_{i_{n}}$; so that $\hat{U}\left(x_{1} \wedge \cdots \wedge x_{n}\right)=U x_{1} \wedge \cdots \wedge U x_{n}$ for all $x_{1}, \ldots, x_{n}$ in $\mathscr{H}$.

Acknowledgment. The authors are grateful to the National Science Foundation (USA) for its support and also to the Mathematics Department of the University of Britsh Columbia for its kind hospitality and the National Research Council of Canada for support during the final stage of this work. 


\section{References}

1. Araki,H.: On the diagonalization of a bilinear Hamiltonian by a Bogoliubov transformation. Publ. Res. Inst. Math. Sci. Kyoto University, Ser. A4, 387-412 (1968)

2. Araki, H.: On quasi-free states of CAR and Bogoliubov automorphisms. Publ. Res. Inst. Math. Sci. Kyoto University, Ser A 6, 385-442 (1970)

3. Balslev,E., Verbeure, A.: States on Clifford algebras. Commun. math. Phys. 7, $55-76$ (1968)

4. Balslev,E., Manuceau,J., Verbeure, A.: Representations of anticommutation relations and Bogoliubov transformations. Commun. math. Phys. 8, 315-326 (1968)

5. Dell'Antonio, G.F.: Structure of the algebras of some free systems. Commun. math. Phys. 9, $81-117(1968)$

6. Emch, G. G.: Algebraic methods in statistical mechanics and quantum field theory. New York: Wiley 1972

7. Haag, R., Kadison, R.V., Kastler, D.: Asymptotic orbits in a free Fermi gas. Commun. math. Phys. 33, 1-22 (1973)

8. Lanford, O., Robinson, D.: Approach to equilibrium of free quantum systems. Commun. math. Phys. 24, 193-210 (1972)

9. Manuceau, J., Rocca, F., Testard, D.: On the product form of quasi-free states. Commun. math. Phys. 12, 43-57 (1969)

10. Manuceau, J., Verbeure,A.: Non-factor quasi-free states of the CAR algebra. Commun. math. Phys. 18, 319-326 (1970)

11. Powers, R.T.: Representations of the canonical anticommutation relations. Thesis Princeton Univ. (1967)

12. Powers, R.T., Størmer,E.: Free states of the canonical anticommutation relations. Commun. math. Phys. 16, 1-33 (1970)

13. Rideau, G.: On some representations of the anticommutation relations. Commun. math. Phys. 9 , 229-241 (1968)

14. Rocca,F., Sirugue, M., Testard,D.: On a class of equilibrium states under the Kubo-MartinSchwinger boundary condition. I. Fermions. Commun. math. Phys. 13, 317-334 (1969)

15. Ruelle,D.: Statistical mechanics. New York-Amsterdam: Benjamin 1969

16. Shale, D., Stinespring, W.F.: States on the Clifford algebra. Ann. Math. 80, 365-381 (1964)

17. Sirugue, M., Winnink, M.: Constraints imposed upon a state of a system that satisfies the K.M.S. boundary condition. Commun. math. Phys. 19, 161-168 (1970)

Communicated by H. Araki

N. M. Hugenholtz

University of Groningen

Institute for Theoretical Physics

P. O. Box 800

Groningen, The Netherlands

R. V. Kadison

Department of Mathematics E 1

University of Pennsylvania

Philadelphia, Pennsylvania, 19174 USA 